\title{
A!
}

This is an electronic reprint of the original article.

This reprint may differ from the original in pagination and typographic detail.

Baroni, Paolo; Kuusi, Tuomo; Lindfors, Casimir; Urbano, José Miguel

\section{Existence and boundary regularity for degenerate phase transitions}

Published in:

SIAM Journal on Mathematical Analysis

DOI:

$10.1137 / 17 \mathrm{M} 1121585$

Published: 01/01/2018

Document Version

Publisher's PDF, also known as Version of record

Please cite the original version:

Baroni, P., Kuusi, T., Lindfors, C., \& Urbano, J. M. (2018). Existence and boundary regularity for degenerate phase transitions. SIAM Journal on Mathematical Analysis, 50(1), 456-490. https://doi.org/10.1137/17M1121585

This material is protected by copyright and other intellectual property rights, and duplication or sale of all or part of any of the repository collections is not permitted, except that material may be duplicated by you for your research use or educational purposes in electronic or print form. You must obtain permission for any other use. Electronic or print copies may not be offered, whether for sale or otherwise to anyone who is not an authorised user. 


\title{
EXISTENCE AND BOUNDARY REGULARITY FOR DEGENERATE PHASE TRANSITIONS*
}

\author{
PAOLO BARONI ${ }^{\dagger}$, TUOMO KUUSI ${ }^{\ddagger}$, CASIMIR LINDFORS $^{\ddagger}$, AND JOSÉ MIGUEL \\ $\mathrm{URBANO}^{\S}$
}

\begin{abstract}
We study the Cauchy-Dirichlet problem associated to a phase transition modeled upon the degenerate two-phase Stefan problem. We prove that weak solutions are continuous up to the parabolic boundary and quantify the continuity by deriving a modulus. As a byproduct, these a priori regularity results are used to prove the existence of a so-called physical solution.
\end{abstract}

Key words. Stefan problem, degenerate equations, intrinsic scaling, boundary modulus of continuity

AMS subject classifications. Primary, 35B65; Secondary, 35A01, 35K65, 80A22

DOI. $10.1137 / 17 \mathrm{M} 1121585$

1. Introduction. In this paper we complete the tour de force, initiated in [1], concerning the regularity of weak solutions for the degenerate $(p \geq 2)$ two-phase Stefan problem $[13,14]$

$$
\begin{cases}\partial_{t}\left[u+H_{0}(u)\right] \ni \operatorname{div}|D u|^{p-2} D u & \text { in } \Omega_{T}, \\ u=g & \text { on } \partial_{p} \Omega_{T},\end{cases}
$$

by proving the continuity up to the boundary. Using this regularity, we also obtain an existence result. Here, $\Omega_{T}:=\Omega \times(0, T]$ denotes the space-time cylinder, with $\Omega \subset \mathbb{R}^{n}, n \geq 2$, a bounded domain, $\partial_{p} \Omega_{T}$ is its parabolic boundary (see subsection 2.1 for the relevant definitions), $H_{0}$ is the Heaviside graph centered at the origin, and $g$ is a continuous boundary datum. The symbol $\ni$ denotes the usual membership in set theory and we stress that the formulation of the (degenerate) Stefan problem we are considering involves a differential inclusion in weak form (see Definition 1.3), since $u+H_{0}(u)$ is a maximal monotone graph.

The outcome of our effort is twofold. On the one hand, we prove sharp a priori estimates for solutions of (1.1) and obtain the boundary continuity, quantified through a modulus, assuming a mild geometric condition on $\Omega$. On the other hand, we use

\footnotetext{
* Received by the editors March 17, 2017; accepted for publication (in revised form) July 27, 2017; published electronically January 30, 2018.

http://www.siam.org/journals/sima/50-1/M112158.html

Funding: The work of the first author was supported by the Gruppo Nazionale per l'Analisi Matematica, la Probabilità e le loro Applicazioni (GNAMPA) of the Istituto Nazionale di Alta Matematica (INdAM). The work of the second and third authors was supported by the Academy of Finland project "Regularity theory for nonlinear parabolic partial differential equations." The third author was also supported by the Vilho, Yrjö, and Kalle Väisälä Foundation. The fourth author was partially supported by CMUC-UID/MAT/00324/2013, funded by the Portuguese government through FCT/MCTES and cofunded by the European Regional Development Fund through partnership agreement PT2020.

${ }^{\dagger}$ Department of Mathematical, Physical, and Computer Sciences, University of Parma, I-43124 Parma, Italy (paolo.baroni@unipr.it).

${ }^{\ddagger}$ Department of Mathematics and Systems Analysis, Aalto University, 00076 Aalto, Finland (tuomo.kuusi@aalto.fi, casmir.lindfors@aalto.fi).

$\S$ Department of Mathematics, CMUC, University of Coimbra, 3001-501 Coimbra, Portugal (jmurb@mat.uc.pt).
} 
this "almost uniform" modulus of continuity at the boundary, together with the interior modulus of continuity we deduced in [1], to build a solution to (1.1), which is continuous up to the boundary and enjoys the same modulus of continuity.

Problem (1.1) when $p=2$ is the celebrated two-phase Stefan problem. The boundary continuity in this case was proven by Ziemer [16] for more general structures, albeit with linear growth with respect to the gradient, but without an explicit, uniform modulus of continuity. This would be provided by DiBenedetto, who, in [5], proved the uniform continuity up to the boundary for solutions to (1.1) (more precisely, for the forthcoming (1.2) for $p=2$, which also takes into account lower order terms) with the modulus of continuity being of iterated logarithmic type in the particular case of the Hölder continuous boundary datum. For an overview on the state of the art for the Stefan problem, we forward the reader to the companion paper [1] and to the survey [13], mainly focusing on viscosity solutions.

Our goal is to extend the result to the degenerate case $p>2$ and to provide, already in the nondegenerate case $p=2$, a more transparent proof of the reduction of the oscillation at the lateral boundary.

More generally, we shall consider in (1.1) the extension

$$
\partial_{t}\left[\beta(u)+H_{a}(\beta(u))\right] \ni \operatorname{div} \mathcal{A}(x, t, u, D u) \quad \text { in } \Omega_{T},
$$

where $\beta$ is a sufficiently smooth function, the Heaviside graph centered at $a \in \mathbb{R}$ is defined by

$$
H_{a}(s)= \begin{cases}0 & \text { if } s<a \\ {[0,1]} & \text { if } s=a \\ 1 & \text { if } s>a\end{cases}
$$

and the vector field $\mathcal{A}$ satisfies the usual $p$-growth conditions (see subsection 1.1 for the exact assumptions). Our first result reads as follows.

THEOREM 1.1. Under the assumptions described in subsection 1.1, given a boundary datum $g \in C\left(\partial_{p} \Omega_{T}\right)$, there exists $u \in C\left(\overline{\Omega_{T}}\right)$ solving the Cauchy-Dirichlet problem for (1.2), in the sense that $u$ is a local weak solution of the equation and $u=g$ on $\partial_{p} \Omega_{T}$. We call $u$ a physical solution.

We remark that the solution we build has the interior modulus of continuity described in [1], where we assumed the existence of a solution built in the way described in this paper. Our other main result concerns a precise modulus of continuity up to the boundary for the physical solution obtained in Theorem 1.1, in the case the regularity of the boundary datum does not overcome a threshold we are going to describe. Let $\omega_{g}$ be a concave modulus of continuity for $g$ :

$$
\sup _{\left(x_{0}, t_{0}\right) \in \partial_{p} \Omega_{T}} \underset{\overline{Q_{r}}\left(x_{0}, t_{0}\right) \cap \partial_{p} \Omega_{T}}{\operatorname{osc}} g \leq \omega_{g}(r) .
$$

Given a point $\left(x_{0}, t_{0}\right) \in \mathbb{R}^{n+1}$ and a radius $r>0, Q_{r}\left(x_{0}, t_{0}\right)$ is the standard (symmetric) parabolic cylinder

$$
Q_{r}\left(x_{0}, t_{0}\right):=B_{r}\left(x_{0}\right) \times\left(t_{0}-r^{p}, t_{0}+r^{p}\right) ;
$$

$\overline{Q_{r}}\left(x_{0}, t_{0}\right)$ is its closure and, for a constant $\varrho>0, Q_{r}^{\varrho}\left(x_{0}, t_{0}\right)$ is the stretched cylinder

$$
Q_{r}^{\varrho}\left(x_{0}, t_{0}\right):=B_{r}\left(x_{0}\right) \times\left(t_{0}-\varrho^{2-p} r^{p}, t_{0}+\varrho^{2-p} r^{p}\right) .
$$


Finally, let us introduce $\bar{q} \equiv \bar{q}(n, p) \geq 2$ as

$$
\bar{q}:= \begin{cases}1+\frac{n}{p} & \text { for } p<n, \\ 2 & \text { for } p \geq n .\end{cases}
$$

We are ready now to state the next theorem.

THEOREM 1.2. Let $u$ be the physical solution of Theorem 1.1, and let $\left(x_{0}, t_{0}\right) \in$ $\partial_{p} \Omega_{T}$ and $R_{0} \in\left(0, r_{\Omega}\right]$ be fixed ( $r_{\Omega}$ will be introduced in (1.10)). Then for every

$$
\alpha \in\left(0, \frac{1}{p^{\prime} \bar{q}}\right)
$$

there exist constants $\vartheta, \lambda_{0}, \tilde{\delta}$ depending on $\alpha$ and the data, such that if we set

$$
\omega(r)=\frac{1}{\vartheta}\left[\frac{1}{\log \left(\log \left(\frac{\lambda_{0} R_{0}}{r}\right)\right)}\right]^{\alpha}
$$

for $r \in\left(0, R_{0}\right]$, and we suppose that

$$
\underset{Q_{R_{0}}^{\tilde{\delta} \omega_{0}}\left(x_{0}, t_{0}\right) \cap \Omega_{T}}{\operatorname{osc}} u \leq \omega_{0} \quad \text { and } \quad \omega_{g}\left(\left(r / R_{0}\right)^{1-\gamma}\right) \leq M \omega(r)
$$

hold for some $\omega_{0}>0, M>0$, and $\gamma \in(0,1)$, then

$$
\underset{Q_{r}\left(x_{0}, t_{0}\right) \cap \Omega_{T}}{\mathrm{OSc}} u \leq c \omega(r)
$$

for all $r \in\left(0, R_{0}\right]$, with $c$ depending on $\gamma, M, R_{0}, \omega_{0}$ and the data.

The previous natural result tells us that once the boundary datum is more regular than the solution, even in the case of smooth $g$, then the solution still has modulus of continuity $\omega$. Clearly, a Hölder continuous function $g$ is an example of a boundary datum satisfying the second condition in (1.8).

1.1. Main assumptions and the concept of solution. Throughout the paper, $\Omega$ is assumed to satisfy the following outer density condition: there exist $\delta \in(0,1)$ and $r_{\Omega}>0$ such that, for $x_{0} \in \partial \Omega$,

$$
\left|B_{r}\left(x_{0}\right) \cap \Omega\right| \leq(1-\delta)\left|B_{r}\left(x_{0}\right)\right| \quad \forall r \in\left(0, r_{\Omega}\right) .
$$

This assumption is standard in this context; see [5, 6]. Ziemer proved quantitative continuity at the boundary assuming the divergence of the Wiener integral (see [16]); although this assumption is weaker than ours, we decided to assume (1.10) to keep the presentation simple, at least in this respect. On the other hand, we stress that the precise form of a Wiener criterion leading to the boundary continuity for the evolutionary $p$-Laplacian is still not clear. The function $\beta: \mathbb{R} \rightarrow \mathbb{R}$ is an increasing $C^{1}$-diffeomorphism satisfying the bi-Lipschitz condition

$$
\Lambda^{-1}|u-v| \leq|\beta(u)-\beta(v)| \leq \Lambda|u-v|,
$$

included, as previously done in $[4,12]$, to account for the thermal properties of the medium, which can change slightly with respect to the temperature. 
The vector field $\mathcal{A}$ is measurable with respect to the first two variables and continuous with respect to the last two, satisfying additionally the following standard growth, coercivity, and monotonicity assumptions:

$$
\begin{gathered}
|\mathcal{A}(x, t, u, \xi)| \leq \Lambda|\xi|^{p-1}, \quad\langle\mathcal{A}(x, t, u, \xi), \xi\rangle \geq \Lambda^{-1}|\xi|^{p}, \\
\langle\mathcal{A}(x, t, u, \xi)-\mathcal{A}(x, t, u, \zeta), \xi-\zeta\rangle>0,
\end{gathered}
$$

for $p \geq 2$, for almost every $(x, t) \in \Omega_{T}$, and for all $(u, \xi, \zeta) \in \mathbb{R} \times \mathbb{R}^{2 n}$, with $\zeta \neq \xi$, for a given constant $\Lambda \geq 1$. It will be useful for future reference to make explicit the modulus of continuity of $\mathcal{A}$ with respect to the last two variables; we suppose that there exist two concave functions $\omega_{\mathcal{A}, u}, \omega_{\mathcal{A}, \xi}:(0, \infty) \rightarrow[0,1]$, such that $\lim _{\rho \searrow 0} \omega_{\mathcal{A}, u}(\rho)=$ $\lim _{\rho \searrow 0} \omega_{\mathcal{A}, \xi}(\rho)=0$, and a function $K:[0, \infty) \times[0, \infty) \rightarrow[1, \infty)$, increasing separately in the two variables, such that

$$
\begin{aligned}
\sup _{(x, t) \in \Omega_{T}}|\mathcal{A}(x, t, u, \xi)-\mathcal{A}(x, t, v, \xi)| & \\
& \leq K(M, \tilde{M})\left[\omega_{\mathcal{A}, u}(|u-v|)+\omega_{\mathcal{A}, \xi}(|\xi-\zeta|)\right]
\end{aligned}
$$

for all $(u, v, \xi, \zeta) \in \mathbb{R}^{2(n+1)}$ such that $|u|+|v| \leq M$ and $|\xi|+|\zeta| \leq \tilde{M}$.

Definition 1.3. A local weak solution of (1.2) is a pair $(u, v)$, with

$$
v \in \beta(u)+H_{a}(\beta(u)),
$$

in the sense of graphs, such that

$$
u \in L_{\mathrm{loc}}^{p}\left(0, T ; W_{\mathrm{loc}}^{1, p}(\Omega)\right) \cap L_{\mathrm{loc}}^{\infty}\left(0, T ; L_{\mathrm{loc}}^{2}(\Omega)\right)=: V_{\mathrm{loc}}^{2, p}\left(\Omega_{T}\right)
$$

and the integral identity

$$
\left.\int_{\mathcal{K}}[v \varphi](\cdot, \tau) d x\right|_{\tau=t_{1}} ^{t_{2}}+\int_{\mathcal{K} \times\left[t_{1}, t_{2}\right]}\left[-v \partial_{t} \varphi+\langle\mathcal{A}(\cdot, \cdot, u, D u), D \varphi\rangle\right] d x d t=0
$$

holds for all $\mathcal{K} \Subset \Omega$ and almost every $t_{1}, t_{2} \in \mathbb{R}$ such that $\left[t_{1}, t_{2}\right] \Subset(0, T]$, and for every test function $\varphi \in L_{\mathrm{loc}}^{p}\left(0, T ; W_{0}^{1, p}(\mathcal{K})\right)$ such that $\partial_{t} \varphi \in L^{2}\left(\mathcal{K} \times\left[t_{1}, t_{2}\right]\right)$.

Remark 1.4. Observe that also $v \in L_{\text {loc }}^{\infty}\left(0, T ; L_{\text {loc }}^{2}(\Omega)\right)$ and that the test functions are in $C\left(\left[t_{1}, t_{2}\right] ; L^{2}(\mathcal{K})\right)$, so every term in (1.14) has a meaning.

1.2. Strategy of the proof. In order to perform a standard reduction of the oscillation, at least in cylinders centered on the lateral boundary, we shall consider three different alternatives. The reduction of the oscillation in the interior has been proven in [1], while at the initial boundary it is a simple consequence of the logarithmic estimate of Lemma 3.5. Let us give a brief and formal description of the structure of the proof. Consider (1.2); clearly we can suppose that the jump is met by the values of the solution in the cylinder considered; otherwise solutions are continuous since they solve $p$-Laplacian-type equations with continuous Cauchy-Dirichlet data. The proof consists in the separate analysis of three alternatives.

Our first alternative (Alt.1) states that the jump is far to the supremum of $u$ on the cylinder. In this case, we can reduce the supremum remaining "above" the jump, and here the equation behaves like the $p$-Laplace equation.

The second alternative (Alt.2) instead means that we are considering the case where the jump is close to the supremum of $u$, and thus it is really influencing the 
behavior of the solution. In this case, we set two further alternatives, (Alt. 2.1) and (Alt.2.2): the latter describes the case where the solution has low energy levels close to the jump for all times (notice the relation between the condition appearing therein and the left-hand side of the energy estimate in (3.2)). Here the equation is still very similar to the $p$-Laplace equation and indeed we reduce the oscillation in $p$-Laplacian-type cylinders. If this is not the case, that is, if the worst-case scenario (Alt. 2.1) happens, solutions are less regular. (Alt. 2.1) encodes the fact that the solution has a high peak of energy close to the jump; in this case, the presence of the jump is significant and therefore the geometry employed must rebalance the further degeneracy it produces.

The implementation of what is described above is quite technical, primarily due to the fact that, as is usual in degenerate evolutionary problems, time scales must depend on the solution itself. We need to define three different time scales to tackle the three different scenarios, and these are not trivial already in the nondegenerate case $p=2$. Moreover, we have to introduce the exponentially small (in terms of the oscillation in the cylinder we are considering) quantity $\widetilde{\omega}$ in (3.3) and this explicitly reflects in the log-log modulus of continuity we obtain.

\section{Preparatory material.}

2.1. Notation. Our notation will be mostly self-explanatory; we mention here some noticeable facts. We shall follow the usual convention of denoting by $c$ a generic constant always greater than or equal to one that may vary from line to line; constants we shall need to recall will be denoted with special symbols, such as $\tilde{c}, c_{*}, c_{\ell}$ or the like. Dependencies of constants will be emphasized between parentheses: $c(n, p, \Lambda)$ will mean that $c$ depends only on $n, p, \Lambda$; often dependencies will be shown right after displays. By saying that a constant depends on the data, we mean that it depends on $n, p, \Lambda, \delta$.

We shall often use the following notational convention: if the display $(n . m)$ contains more than one element, then we shall denote with $(\text { n.m })_{j}$ its $j$ th element. As significant examples, with $(1.1)_{1}$ we shall refer to the differential inclusion and with $(1.1)_{2}$ to the associated Cauchy-Dirichlet condition; $(1.8)_{1}$ will be the first assumption described in (1.8), (3.31) $)_{2}$ will be the definition of $T^{4}$, and so on.

By parabolic boundary of a cylinder $\mathcal{K}:=C \times \Gamma$, we shall mean $\partial_{p} \mathcal{K}:=(\bar{C} \times$ $\{\inf \Gamma\}) \cup(\partial C \times \Gamma)$. Its lateral boundary will be denoted as $\partial_{\text {lat }} \mathcal{K}:=\partial C \times \Gamma$ and its initial boundary $\bar{C} \times\{\inf \Gamma\}$ will be $\partial_{\text {ini }} \mathcal{K}$. We denote by $(f)_{A}$ the averaged integral

$$
(f)_{A}:=f_{A} f(\xi) d \xi:=\frac{1}{|A|} \int_{A} f(\xi) d \xi
$$

where $A \in \mathbb{R}^{k}$ is a measurable set with $0<|A|<\infty$ and $f: A \rightarrow \mathbb{R}^{m}$ an integrable map, with $k, m \geq 1$. Finally we stress that with the statement "a vector field with the same structure as $\mathcal{A}$ " (or "structurally similar to $\mathcal{A}$," or similar expressions) we shall mean that the vector field will satisfy (1.12), eventually with $\Lambda$ replaced by a constant depending only on $n, p, \Lambda$, and continuous with respect to the last two variables. $\mathbb{N}$ is the set $\{1,2, \ldots\}$, while $\mathbb{N}_{0}:=\mathbb{N} \cup\{0\}$.

2.2. Approximation of the problem. Let $\rho_{\varepsilon}$ be the standard symmetric, positive, one-dimensional mollifier, supported in $(-\varepsilon, \varepsilon)$, obtained via rescaling of $\rho \in C_{c}^{\infty}(-1,1)$. We set

$$
H_{a, \varepsilon}(s):=\left(\rho_{\varepsilon} * H_{a}\right)(s), \quad s \in \mathbb{R}
$$


and observe that $H_{a, \varepsilon}$ is smooth and

$$
\operatorname{supp} H_{a, \varepsilon}^{\prime} \subset(a-\varepsilon, a+\varepsilon), \quad \int_{\mathbb{R}} H_{a, \varepsilon}^{\prime}(v) d v=1 .
$$

Those will be the unique properties of $H_{a, \varepsilon}$ we will use in the proofs of section 3 (actually, we use the fact that the integral is bounded from above by one). Let $u_{\varepsilon}$ solve the approximate Cauchy-Dirichlet problem

$$
\begin{cases}\partial_{t}\left[\beta\left(u_{\varepsilon}\right)+H_{a, \varepsilon}\left(\beta\left(u_{\varepsilon}\right)\right)\right]-\operatorname{div} \mathcal{A}\left(x, t, u_{\varepsilon}, D u_{\varepsilon}\right)=0 & \text { in } \Omega_{T}, \\ u_{\varepsilon}=g & \text { on } \partial_{p} \Omega_{T} .\end{cases}
$$

Setting

$$
w_{\varepsilon}:=\beta\left(u_{\varepsilon}\right), \quad w_{0}:=\beta(g),
$$

we arrive at the regularized Cauchy-Dirichlet problem

$$
\begin{cases}\partial_{t} w_{\varepsilon}-\operatorname{div} \overline{\mathcal{A}}\left(x, t, w_{\varepsilon}, D w_{\varepsilon}\right)=-\partial_{t} H_{a, \varepsilon}\left(w_{\varepsilon}\right) & \text { in } \Omega_{T} \\ w_{\varepsilon}=w_{0} & \text { on } \partial_{p} \Omega_{T}\end{cases}
$$

where

$$
\overline{\mathcal{A}}(x, t, \mu, \xi):=\mathcal{A}\left(x, t, \beta^{-1}(\mu),\left[\beta^{\prime}\left(\beta^{-1}(\mu)\right)\right]^{-1} \xi\right),
$$

for a.e. $(x, t) \in \Omega_{T},(\mu, \xi) \in \mathbb{R} \times \mathbb{R}^{n}$. Observe that the growth and ellipticity bounds for $\overline{\mathcal{A}}$ are inherited from $\mathcal{A}$ and from the two-sided bound for $\beta^{\prime}$ : indeed

$$
|\overline{\mathcal{A}}(x, t, \mu, \xi)| \leq \Lambda^{p}|\xi|^{p-1}, \quad\langle\overline{\mathcal{A}}(x, t, \mu, \xi), \xi\rangle \geq \Lambda^{-p}|\xi|^{p}
$$

hold for almost every $(x, t) \in \Omega_{T}$ and for all $(\mu, \xi) \in \mathbb{R} \times \mathbb{R}^{n}$. Moreover, $\overline{\mathcal{A}}$ is clearly continuous with respect to the last two variables since $\beta$ is a $C^{1}$-diffeomorphism.

By standard regularity theory for degenerate parabolic equations (see $[6,11,15]$ ), we have that the solution $w_{\varepsilon}$ of $(2.4)_{1}$ is Hölder continuous since $\beta\left(u_{\varepsilon}\right)+H_{a, \varepsilon}\left(\beta\left(u_{\varepsilon}\right)\right)$ is now a diffeomorphism. This kind of regularity depends, however, on the regularization and as such it will deteriorate as $\varepsilon \downarrow 0$. Nonetheless, we may assume that the solution of the regularized equation is continuous, having, in particular, pointwise values. Sometimes we will use the compact notation

$$
\mathcal{H}(s):=s+H_{a, \varepsilon}(s) .
$$

2.3. Scaling of the equation. It will be useful later on to rescale the solution of (2.4) in the following way: define, for $\lambda \geq 1$,

$$
\hat{v}(y, \tau):=\frac{w_{\varepsilon}\left(y, t_{0}+\lambda^{2-p}\left(\tau-t_{0}\right)\right)}{\lambda}
$$

in $E=\Omega \times\left(t_{0}-\lambda^{p-2} t_{0}, t_{0}+\lambda^{p-2}\left(T-t_{0}\right)\right]$. If we set

$$
\hat{H}(s):=\frac{H_{a, \varepsilon}(\lambda s)}{\lambda}=\frac{H_{a / \lambda, \varepsilon / \lambda}(s)}{\lambda}, \quad \hat{g}(y, \tau):=\frac{w_{0}\left(y, t_{0}+\lambda^{2-p}\left(\tau-t_{0}\right)\right)}{\lambda},
$$

Copyright (c) by SIAM. Unauthorized reproduction of this article is prohibited. 
it is then easy to see that $\hat{v}$ solves the Cauchy-Dirichlet problem

$$
\begin{cases}\partial_{\tau} \hat{v}-\operatorname{div}_{y} \hat{\mathcal{A}}(y, \tau, \hat{v}, D \hat{v})=-\partial_{\tau} \hat{H}(\hat{v}) & \text { in } E, \\ \hat{v}=\hat{g} & \text { on } \partial_{p} E,\end{cases}
$$

with $\hat{\mathcal{A}}(y, \tau, \mu, \xi):=\overline{\mathcal{A}}(y, \tau, \lambda \mu, \lambda \xi) / \lambda^{p-1}$ having the same structural properties as $\mathcal{A}$. Note that in particular we have

$$
\operatorname{supp} \hat{H}^{\prime} \subset\left(\frac{a-\varepsilon}{\lambda}, \frac{a+\varepsilon}{\lambda}\right) \quad \text { and } \quad \int_{\mathbb{R}} \hat{H}^{\prime}(\sigma) d \sigma \leq 1 .
$$

2.4. Sobolev's inequalities. We recall here, in a unified and slightly formal setting, some parabolic Sobolev-type inequalities that will be useful in the rest of the paper. To start with, we recall that we can denote the Sobolev conjugate exponent of $p$ as $p^{*}=\kappa p$, where

$$
\kappa:= \begin{cases}\frac{n}{n-p} & \text { for } p<n, \\ \text { any number }>1 & \text { for } p=n, \\ +\infty & \text { for } p>n\end{cases}
$$

For a ball $B$ in $\mathbb{R}^{n}$ and an interval $\Gamma$ of $\mathbb{R}$, we consider functions

$$
\begin{aligned}
& w \in L^{p}\left(\Gamma ; W^{1, p}(B)\right) \cap L^{\infty}\left(\Gamma ; L^{2}(B)\right) \\
& \quad \text { and } \quad \phi \in C^{\infty}(B \times \Gamma), \quad \phi(\cdot, \tau) \in C_{c}^{\infty}(B) \quad \forall \tau \in \Gamma ;
\end{aligned}
$$

applying Hölder's inequality with respect to the time variable with conjugate exponents $\kappa, \kappa^{\prime}$, and afterward the standard Sobolev's inequality slicewise for functions in $W_{0}^{1, p}(B)$, we infer

$$
\begin{aligned}
& f_{B \times \Gamma} w^{2(1-1 / \kappa)+p} \phi^{p(2-1 / \kappa)} d x d t \\
\leq & c(n, p)|B|^{p / n}|\Gamma|^{1-1 / \kappa}\left[\frac{1}{|\Gamma|} \sup _{\tau \in \Gamma} f_{B}\left[w^{2} \phi^{p}\right](\cdot, \tau) d x\right]^{1-1 / \kappa} f_{B \times \Gamma}|D(w \phi)|^{p} d x d t .
\end{aligned}
$$

From now on, we shall make use of the formal agreement that when $\kappa=\infty$, then $1 / \kappa=0, \kappa /(\kappa-1)=1$ and

$$
\left[f_{B}(w \phi)^{\kappa p} d x\right]^{1 / \kappa}:=\|w \phi\|_{L^{\infty}(B)}^{p}
$$

note that in this case there is no necessity to apply Hölder's inequality.

Finally, once chosen a number $\alpha$ as in (1.6) and, setting $q=\frac{1}{p^{\prime} \alpha}>\bar{q}$, with $\bar{q}$ defined in (1.5), we fix $\kappa \equiv \kappa(q) \equiv \kappa(p, \alpha)$, in the case $p=n$, as

$$
\kappa=\frac{q}{q-2}>1
$$

in the rest of the paper we shall implicitly keep $\kappa$ fixed with this value. This, in view of the fact that the lower bound for $q$ satisfies the (formal when $\kappa=\infty$ ) relation $\bar{q}=1+\kappa /(\kappa-1)$, ensures that

$$
\left(1-\frac{1}{q}\right)\left(2-\frac{1}{\kappa}\right)>1 .
$$

Copyright $\odot$ by SIAM. Unauthorized reproduction of this article is prohibited. 
3. Reducing the oscillation at the boundary. In this section we shall consider a function $v$ solving

$$
\begin{cases}\partial_{t} v-\operatorname{div} \widetilde{\mathcal{A}}(x, t, v, D v)=-\partial_{t} H_{b, \varepsilon}(v) & \text { in } \Omega_{T}, \\ v=\tilde{g} & \text { on } \partial_{p} \Omega_{T}\end{cases}
$$

with the Cauchy-Dirichlet datum $\tilde{g}$ being a uniformly continuous function and $\widetilde{\mathcal{A}}$ satisfying $(1.12)_{1,2}$. By regularity theory for evolutionary $p$-Laplace-type equations (see $[6,15])$, we actually have that the solution $v$ is continuous up to the boundary since $\sigma \mapsto \sigma+H_{b, \varepsilon}(\sigma)$ is a diffeomorphism for $\varepsilon>0$ fixed. Later on we shall take as $v$ the function $w_{\varepsilon}$ appearing in (2.3), conveniently rescaled (and this explains the fact that the jump happens at $s=b \neq a$ ), and as $\tilde{g}$ the boundary datum $w_{0}$, also rescaled.

As the first result we have the following Caccioppoli's inequality at the boundary.

Lemma 3.1. Let $v$ be a solution to (3.1) and let $Q=B \times \Gamma$ be a cylinder such that $Q \cap \partial_{p} \Omega_{T} \neq \emptyset$. Then there exists a constant $c$ depending on $p$ and $\Lambda$ such that

$$
\begin{aligned}
& \sup _{\tau \in \Gamma \cap(0, T)} \frac{1}{|\Gamma \cap(0, T)|} f_{B \cap \Omega}\left[\int_{k}^{v} H_{b, \varepsilon}^{\prime}(\xi)(\xi-k)_{+} d \xi \phi^{p}\right](\cdot, \tau) d x \\
&+\sup _{\tau \in \Gamma \cap(0, T)} \frac{1}{|\Gamma \cap(0, T)|} f_{B \cap \Omega}\left[(v-k)_{+}^{2} \phi^{p}\right](\cdot, \tau) d x+f_{Q \cap \Omega_{T}}\left|D(v-k)_{+} \phi\right|^{p} d x d t \\
& \leq c f_{Q \cap \Omega_{T}}\left[(v-k)_{+}^{p}|D \phi|^{p}+(v-k)_{+}^{2}\left(\partial_{t} \phi^{p}\right)_{+}\right] d x d t \\
& \quad+c f_{Q \cap \Omega_{T}} \int_{k}^{v} H_{b, \varepsilon}^{\prime}(\xi)(\xi-k)_{+} d \xi\left(\partial_{t} \phi^{p}\right)_{+} d x d t
\end{aligned}
$$

for any $k>\sup _{\bar{Q} \cap \partial_{p} \Omega_{T}} \tilde{g}$ and any test function $\phi \in C^{\infty}(Q)$ vanishing on $\partial_{p} Q$.

Proof. In order to get (3.2), we test the local weak formulation of (3.1) with $\varphi=(v-k)_{+} \phi^{p}$; notice that $\varphi$ has a compact support in $\Omega_{T}$, since $v$ is continuous up to the boundary as it solves the regularized equation. The calculations are now standard and we refer to [1, Lemma 2.1] or [4].

Remark 3.2. Note that it makes sense to apply the Sobolev's inequality of (2.10) to functions of the form $\varphi:=(v-k)_{+} \phi, \phi \in C_{c}^{\infty}(B), k \in \mathbb{R}$ large as in Lemma 3.1, on balls centered on the lateral boundary of $\Omega_{T}$, just setting $\varphi \equiv 0$ outside $\Omega_{T}$. In view of (1.10), taking averages in (2.10) with respect to $B$ is equivalent to taking them with respect to $B \cap \Omega$, so there will not be any possible misunderstanding. Another occurrence when we shall apply Sobolev's inequality $(2.10)$ is when $\phi(\cdot, \tau)=0$ in $\mathbb{R}^{n} \backslash \Omega$ for almost every $\tau \in \Gamma$; in this case, we have

$$
|B \cap\{\phi(\cdot, \tau)=0\}| \geq \delta|B| \quad \text { for a.e. } \tau
$$

by our density assumption (1.10), and again a classic Sobolev-type inequality (see [9, Theorem 1, p. 189]) leads to (2.10), with the constant also depending on $\delta$. Also a Poincaré's inequality is available in this case (see, for instance, (3.24)).

3.1. Reducing the oscillation at the lateral boundary. Assume now that $\left(x_{0}, t_{0}\right) \in \partial_{\mathrm{lat}} \Omega_{T}$ and recall that $\Omega$ satisfies the outer density condition (1.10) with 
parameters $\delta \in(0,1)$ and $r_{\Omega}>0$. Let $\omega \in(0,1]$ and define the following auxiliary number for $\varepsilon_{1} \in(0,1)$ to be fixed later:

$$
\widetilde{\omega}=\varepsilon_{1} \omega \exp \left(-\left[\varepsilon_{1} \omega\right]^{-p^{\prime} q}\right)<\frac{1}{2} \varepsilon_{1} \omega<\omega .
$$

We shall need to work with the two time scales $T^{1}:=\left[\varepsilon_{1} \omega\right]^{2-p} r^{p}, T^{3}:=\widetilde{\omega}^{1-p} r^{p}$ in order to handle the degeneracy given by the jump. Moreover we shall also need the scale $T^{2}:=\left[\varepsilon_{2} \widetilde{\omega}\right]^{2-p} r^{p}, \varepsilon_{2} \in(0,1)$, when away from the jump, i.e., when dealing with the degeneracy given only by the $p$-Laplacian operator; see subsection 3.1.3. We shall moreover always consider $\varepsilon_{1} \leq \varepsilon_{2}^{p-2}$ (see (3.27)); in view of this, (3.3), and the trivial fact that $\widetilde{\omega} \leq \varepsilon_{1}$, we have

$$
T^{1}=\left[\varepsilon_{1} \omega\right]^{2-p} r^{p} \leq \widetilde{\omega}^{2-p} r^{p} \leq T^{2}=\varepsilon_{2}^{2-p} \widetilde{\omega}^{1+(1-p)} r^{p} \leq \widetilde{\omega}^{1-p} r^{p}=T^{3} .
$$

We also define for $\sigma>0$ the cylinders

$$
\sigma Q^{i}:=\left(B_{\sigma r}\left(x_{0}\right) \times\left(t_{0}-\sigma T^{i}, t_{0}\right)\right) \cap \Omega_{T}, \quad i=1,2,3 .
$$

Note that clearly $Q^{1} \subset Q^{2} \subset Q^{3}$.

From now on we shall write

$$
\mu^{+}:=\sup _{Q^{3}} v, \quad \mu^{-}:=\inf _{Q^{3}} v .
$$

We further assume that

$$
b \in\left[\mu^{-}, \mu^{+}\right]
$$

and

$$
\sup _{\bar{Q}^{3} \cap \partial_{p} \Omega_{T}} \tilde{g} \leq \mu^{+}-\frac{\omega}{8}, \quad \varepsilon \leq \frac{\widetilde{\omega}}{2} .
$$

We consider two cases: either the jump is close to the supremum of $v$,

$$
b \leq \mu^{+}-2 \widetilde{\omega}
$$

or this does not hold,

$$
b>\mu^{+}-2 \widetilde{\omega} .
$$

In the case of (Alt.2), we consider two further alternatives: either

$$
\sup _{\max \left\{0, t_{0}-\frac{1}{4} T^{1}\right\}<t<t_{0}} f_{B_{r / 4} \cap \Omega} \int_{\mu^{+}-3 \widetilde{\omega}}^{v(\cdot, t)} H_{b, \varepsilon}^{\prime}(\xi) d \xi d x>\varepsilon_{3}^{-1}\left[\varepsilon_{1} \omega\right]^{q}
$$

is in force or the converse inequality

$$
\sup _{\max \left\{0, t_{0}-\frac{1}{4} T^{1}\right\}<t<t_{0}} f_{B_{r / 4} \cap \Omega} \int_{\mu^{+}-3 \widetilde{\omega}}^{v(\cdot, t)} H_{b, \varepsilon}^{\prime}(\xi) d \xi d x \leq \varepsilon_{3}^{-1}\left[\varepsilon_{1} \omega\right]^{q}
$$

holds, where $q$ satisfies $(1.5)$ and $\varepsilon_{3} \in(0,1)$ will be chosen later. Note that it would be equivalent (see (Alt. 1) and (3.6) $)_{2}$ and consider also (2.2)) to put as the lower bound in the integral of $H_{b, \varepsilon}^{\prime}$ the point $b-\varepsilon$; we keep this choice also to meet the formal explanation in subsection 1.2.

Copyright $@$ by SIAM. Unauthorized reproduction of this article is prohibited. 
3.1.1. Strategy of the proof revisited. There are three free parameters $\varepsilon_{1}, \varepsilon_{2}$, $\varepsilon_{3}$ appearing above. The strategy for choosing them is to first fix $\varepsilon_{2}$ in the case (Alt. 1); this choice is independent of $\varepsilon_{1}$ and $\varepsilon_{3}$. We subsequently fix $\varepsilon_{3}$ in the analysis of (Alt. 2) and (Alt. 2.1) (see (3.26)), independently of $\varepsilon_{1}$ and $\varepsilon_{2}$, and finally, $\varepsilon_{1}$ is chosen to depend on the data and $\varepsilon_{2}, \varepsilon_{3}$ while analyzing the cases (Alt. 2) and (Alt. 2.2) (see $(3.27))$.

LEMma 3.3. Suppose that $v$ is a weak solution to (3.1) satisfying (3.5), (3.6) and suppose that $\varepsilon_{1}, \varepsilon_{2}$ are small enough $\left(\varepsilon_{1}, \varepsilon_{2} \leq 2^{-10}\right)$. Then there is a constant $c_{\ell} \equiv c_{\ell}(n, p, \Lambda, \delta) \geq 1$ such that the following holds:

- if $v$ satisfies the first alternative (Alt. 1), then

$$
\frac{\left|\frac{1}{8} Q^{2} \cap\left\{v>\mu^{+}-2 \varepsilon_{2} \widetilde{\omega}\right\}\right|}{\left|\frac{1}{8} Q^{2}\right|} \leq \frac{c_{\ell}}{\left[\log \left(1 / \varepsilon_{2}\right)\right]^{1 / p^{\prime}}} ;
$$

- if $v$ satisfies the second alternative (Alt.2), then

$$
\frac{\left|\frac{1}{2} Q^{3} \cap\left\{v>\mu^{+}-8 \widetilde{\omega}\right\}\right|}{\left|\frac{1}{2} Q^{3}\right|} \leq c_{\ell}\left[\varepsilon_{1} \omega\right]^{q} ;
$$

- if $v$ satisfies the second alternative (Alt.2) and also (Alt.2.2), then

$$
\frac{\left|\frac{1}{8} Q^{1} \cap\left\{v>\mu^{+}-2 \varepsilon_{1} \omega\right\}\right|}{\left|\frac{1}{8} Q^{1}\right|} \leq \frac{c_{\ell} \varepsilon_{3}^{-1 / p}}{\left[\log \left(1 / \varepsilon_{1}\right)\right]^{1 / p^{\prime}}} .
$$

Proof. Let us first prove (3.9). We define

$$
k_{j}:=\mu^{+}-2^{-j} \omega, \quad w_{j}:=\left(v-k_{j}\right)_{+}, \quad \widehat{w}_{j}:=\min \left\{w_{j}, k_{j+1}-k_{j}\right\}
$$

for all $3 \leq j \leq \bar{\jmath}$, where $\bar{\jmath}$ is the integer satisfying

$$
2^{-(\bar{\jmath}+2)}<2 \varepsilon_{1} \leq 2^{-(\bar{\jmath}+1)} .
$$

By $(3.6)_{1}$ we have that for all $j \geq 3$

$$
k_{j} \geq \mu^{+}-\frac{\omega}{8} \geq \sup _{Q^{3} \cap \partial_{p} \Omega_{T}} \tilde{g} \geq \sup _{Q^{1} \cap \partial_{p} \Omega_{T}} \tilde{g}
$$

therefore, $w_{j}(\cdot, t)$ vanishes in a neighborhood of $\partial \Omega$ for every $t \in\left(t_{0}-T^{1}, t_{0}\right)$. Thus we may extend it to be zero outside of $\Omega$ in such a way that

$$
\hat{w}_{j} \in L^{p}\left(t_{0}-T^{1}, t_{0} ; W^{1, p}\left(B_{r}\left(x_{0}\right)\right)\right)
$$

The density condition (1.10) readily implies that

$$
\left|B_{r / 8}\left(x_{0}\right) \cap\left\{\widehat{w}_{j}(\cdot, t)=0\right\}\right| \geq \delta\left|B_{r / 8}\left(x_{0}\right)\right|
$$

for all $t \in\left(t_{0}-T^{1}, t_{0}\right)$. Using this condition we have by the standard application of the Poincaré inequality that

$$
\int_{B_{r / 8}} \widehat{w}_{j}(\cdot, t) d x \leq c(n, \delta) r \int_{B_{r / 8}}\left|D \widehat{w}_{j}(\cdot, t)\right| d x
$$

Copyright $@$ by SIAM. Unauthorized reproduction of this article is prohibited. 
for every $t \in\left(t_{0}-T^{1}, t_{0}\right)$. Now we integrate the previous inequality over $\left(t_{0}-\frac{1}{8} T^{1}, t_{0}\right)$ and then estimate from below the left-hand side in the following way:

$$
\int_{\frac{1}{8} Q^{1}} \widehat{w}_{j} d x d t \geq\left(k_{j+1}-k_{j}\right)\left|\frac{1}{8} Q^{1} \cap\left\{v \geq k_{j+1}\right\}\right|=2^{-(j+1)} \omega\left|\frac{1}{8} Q^{1} \cap\left\{v \geq k_{j+1}\right\}\right| .
$$

By Hölder's inequality we bound from above

$$
\int_{\frac{1}{8} Q^{1}}\left|D \widehat{w}_{j}\right| d x d t \leq\left[\int_{\frac{1}{8} Q^{1}}\left|D w_{j}\right|^{p} d x d t\right]^{1 / p}\left|\frac{1}{8} Q^{1} \cap\left\{k_{j}<v<k_{j+1}\right\}\right|^{1 / p^{\prime}} .
$$

Combining the above displays leads to

$$
\begin{aligned}
\left|\frac{1}{8} Q^{1} \cap\left\{v \geq k_{j+1}\right\}\right| \leq c(n, \delta)\left|\frac{1}{8} Q^{1}\right|^{1 / p}\left[r^{p}\left[2^{-j} \omega\right]^{-p}\right. & \left.f_{\frac{1}{8} Q^{1}}\left|D\left(v-k_{j}\right)_{+}\right|^{p} d x d t\right]^{1 / p} \\
& \times\left|\frac{1}{8} Q^{1} \cap\left\{k_{j}<v<k_{j+1}\right\}\right|^{1 / p^{\prime}} .
\end{aligned}
$$

At this point we want to use the boundary Caccioppoli's inequality, Lemma 3.1, with $Q=\frac{1}{4} Q^{1}, k=k_{j}$, and $\phi \in C^{\infty}\left(\frac{1}{4} Q^{1}\right)$ a standard cutoff function vanishing on the parabolic boundary with $0 \leq \phi \leq 1, \phi \equiv 1$ on $\frac{1}{8} Q^{1}$, and

$$
\left|\partial_{t} \phi^{p}\right| \leq \frac{c(p)}{T^{1}}, \quad|D \phi| \leq \frac{c}{r} .
$$

Observing that by (3.11) we have for any $j \leq \bar{\jmath}$

$$
T^{1}=\left[\varepsilon_{1} \omega\right]^{2-p} r^{p} \geq\left[2^{-(j+2)} \omega\right]^{2-p} r^{p},
$$

and after some simple algebraic manipulations we obtain

$$
\begin{aligned}
f_{\frac{1}{8} Q^{1}}\left|D\left(v-k_{j}\right)_{+}\right|^{p} d x d t & \leq \frac{c}{r^{p}}\left[f_{\frac{1}{4} Q^{1}}\left(\left(v-k_{j}\right)_{+}^{p}+\left(v-k_{j}\right)_{+}^{2}\left[2^{-j} \omega\right]^{p-2}\right) d x d t\right. \\
& \left.+\left[2^{-j} \omega\right]^{p-2} f_{\frac{1}{4} Q^{1}} \int_{k_{j}}^{v} H_{b, \varepsilon}^{\prime}(\xi)\left(\xi-k_{j}\right)_{+} d \xi d x d t\right]
\end{aligned}
$$

Now we have to use (Alt. 2.2): we can estimate using $\left(v-k_{j}\right)_{+} \leq 2^{-j} \omega$ and the facts that $b-\varepsilon>\mu^{+}-3 \widetilde{\omega}$ and $H_{b, \varepsilon}^{\prime}(\xi)=0$ whenever $\xi<b-\varepsilon$,

$$
\int_{k_{j}}^{v} H_{b, \varepsilon}^{\prime}(\xi)\left(\xi-k_{j}\right)_{+} d \xi \leq 2^{-j} \omega \int_{\mu^{+}-3 \widetilde{\omega}}^{v} H_{b, \varepsilon}^{\prime}(\xi) d \xi
$$

Then, by (Alt. 2.2) we infer

$$
\begin{aligned}
{\left[2^{-j} \omega\right]^{p-2} f_{\frac{1}{4} Q^{1}} \int_{k_{j}}^{v} } & H_{b, \varepsilon}^{\prime}(\xi)\left(\xi-k_{j}\right)_{+} d \xi d x d t \\
& \leq\left[2^{-j} \omega\right]^{p-1} \sup _{\max \left\{0, t_{0}-\frac{1}{4} T^{1}\right\}<t<t_{0}} f_{B_{r / 4} \cap \Omega} \int_{\mu^{+}-3 \widetilde{\omega}}^{v(\cdot, t)} H_{b, \varepsilon}^{\prime}(\xi) d \xi d x \\
& \leq \varepsilon_{3}^{-1}\left[2^{-j} \omega\right]^{p}
\end{aligned}
$$

Copyright (c) by SIAM. Unauthorized reproduction of this article is prohibited. 
since $\varepsilon_{1} \leq 2^{-j}$ by (3.11) and $q>1$. It follows by combining (3.17), (3.18), and (3.19) that

$$
\left|\frac{1}{8} Q^{1} \cap\left\{v \geq k_{j+1}\right\}\right| \leq c \varepsilon_{3}^{-1 / p}\left|\frac{1}{8} Q^{1}\right|^{1 / p}\left|\frac{1}{8} Q^{1} \cap\left\{k_{j}<v<k_{j+1}\right\}\right|^{1 / p^{\prime}} .
$$

Taking the power $p^{\prime}$ from both sides and then summing up for $j=3, \ldots, \bar{\jmath}$ gives

$$
\begin{aligned}
(\bar{\jmath}-2)\left|\frac{1}{8} Q^{1} \cap\left\{v \geq k_{\bar{\jmath}+1}\right\}\right|^{p^{\prime}} & \leq c \varepsilon_{3}^{-p^{\prime} / p}\left|\frac{1}{8} Q^{1}\right|^{p^{\prime} / p} \sum_{j=3}^{\bar{\jmath}}\left|\frac{1}{8} Q^{1} \cap\left\{k_{j}<v<k_{j+1}\right\}\right| \\
& \leq c \varepsilon_{3}^{-p^{\prime} / p}\left|\frac{1}{8} Q^{1}\right|^{1 /(p-1)+1}=c \varepsilon_{3}^{-p^{\prime} / p}\left|\frac{1}{8} Q^{1}\right|^{p^{\prime}}
\end{aligned}
$$

and hence, finally,

$$
\frac{\left|\frac{1}{8} Q^{1} \cap\left\{v \geq \mu^{+}-2^{-(\bar{\jmath}+1)} \omega\right\}\right|}{\left|\frac{1}{8} Q^{1}\right|} \leq \frac{c \varepsilon_{3}^{-1 / p}}{(\bar{\jmath}-2)^{1 / p^{\prime}}}
$$

with $c$ depending on $n, p, \Lambda, \delta$. The result now follows easily, since $-5 \geq\left(\log _{2} \varepsilon_{1}\right) / 2$ implies

$$
\bar{\jmath}-2 \geq-\log _{2} \varepsilon_{1}-5 \geq-c \log \varepsilon_{1} .
$$

We come to the proof of (3.8). The levels $k_{j}$ and the functions $w_{j}, \hat{w}_{j}$ are defined exactly as in (3.10) for $3 \leq j \leq \bar{\jmath}$, but this time with $\bar{\jmath}$ being the integer satisfying

$$
2^{-(\bar{\jmath}+2)} \omega<8 \varepsilon_{1} \exp \left(-\left[\varepsilon_{1} \omega\right]^{-p^{\prime} q}\right) \omega=8 \widetilde{\omega} \leq 2^{-(\bar{\jmath}+1)} \omega ;
$$

again this yields $\widetilde{\omega} \leq 2^{-j} \omega$ for all $j \leq \bar{\jmath}$. Now we can proceed similarly as above, since (3.12) still clearly holds. Extending again $\hat{w}_{j}$ to zero outside $\Omega$ in such a way that $\hat{w}_{j} \in L^{p}\left(t_{0}-T^{3}, t_{0} ; W^{1, p}\left(B_{r}\left(x_{0}\right)\right)\right)$, we have (3.13) over $B_{r / 2}$ for all $t \in\left(t_{0}-T^{3}, t_{0}\right)$ and hence (3.14) in $\left(t_{0}-T^{3}, t_{0}\right)$. Integrating and again estimating from below the left-hand side as in (3.15) and the right-hand side as in (3.16) yields

$$
2^{-j} \omega\left|\frac{1}{2} Q^{3} \cap\left\{v \geq k_{j+1}\right\}\right| \leq c\left[r^{p} \int_{\frac{1}{2} Q^{3}}\left|D w_{j}\right|^{p} d x d t\right]^{1 / p}\left|\frac{1}{2} Q^{3} \cap\left\{k_{j}<v<k_{j+1}\right\}\right|^{1 / p^{\prime}}
$$

with $c \equiv c(n, \delta)$. Now, by the choice of $\bar{\jmath}$, we have for any $j \leq \bar{\jmath}$ that $T^{3}=\widetilde{\omega}^{1-p} r^{p} \geq$ $\left[2^{-j} \omega\right]^{1-p} r^{p}$. Thus the boundary Caccioppoli's inequality in this case takes the form

$$
\begin{aligned}
f_{\frac{1}{2} Q^{3}}\left|D\left(v-k_{j}\right)_{+}\right|^{p} d x d t \leq \frac{c}{r^{p}}\left[f_{Q^{3}}\right. & \left(\left(v-k_{j}\right)_{+}^{p}+\left(v-k_{j}\right)_{+}^{2}\left[2^{-j} \omega\right]^{p-1}\right) d x d t \\
& \left.+\left[2^{-j} \omega\right]^{p-1} f_{Q^{3}} \int_{k_{j}}^{v} H_{b, \varepsilon}^{\prime}(\xi)\left(\xi-k_{j}\right)_{+} d \xi d x d t\right]
\end{aligned}
$$

Now, recalling that $\left(v-k_{j}\right)_{+} \leq 2^{-j} \omega$, we simply estimate by $(2.2)_{2}$

$$
\int_{k_{j}}^{v} H_{b, \varepsilon}^{\prime}(\xi)\left(\xi-k_{j}\right)_{+} d \xi \leq\left(v-k_{j}\right)_{+} \int_{\mathbb{R}} H_{b, \varepsilon}^{\prime}(\xi) d \xi \leq 2^{-j} \omega
$$

and this leads to

$$
f_{\frac{1}{2} Q^{3}}\left|D\left(v-k_{j}\right)_{+}\right|^{p} d x d t \leq \frac{c}{r^{p}}\left[2^{-j} \omega\right]^{p}
$$

Copyright $@$ by SIAM. Unauthorized reproduction of this article is prohibited. 
This is to say, the choice of the time scale $T^{3}$ is sufficient to rebalance the inequality. We obtain again

$$
\left|\frac{1}{2} Q^{3} \cap\left\{v \geq k_{j+1}\right\}\right| \leq c\left|\frac{1}{2} Q^{3}\right|^{1 / p}\left|\frac{1}{2} Q^{3} \cap\left\{k_{j}<v<k_{j+1}\right\}\right|^{1 / p^{\prime}},
$$

and as above, after summing up for $j=3, \ldots, \bar{\jmath}$ gives

$$
(\bar{\jmath}-2)^{1 / p^{\prime}}\left|\frac{1}{2} Q^{3} \cap\left\{v \geq \mu^{+}-8 \widetilde{\omega}\right\}\right| \leq(\bar{\jmath}-2)^{1 / p^{\prime}}\left|\frac{1}{2} Q^{3} \cap\left\{v \geq k_{\bar{\jmath}+1}\right\}\right| \leq c\left|\frac{1}{2} Q^{3}\right| .
$$

We again conclude by estimating

$$
2^{-(\bar{\jmath}+2)} \leq 8 \varepsilon_{1} \exp \left(-\left[\varepsilon_{1} \omega\right]^{-p^{\prime} q}\right) \leq 2^{-7-\left[\varepsilon_{1} \omega\right]^{-p^{\prime} q}}
$$

since $\varepsilon_{1} \leq 2^{-10}$ and thus

$$
\bar{\jmath}-2 \geq\left[\varepsilon_{1} \omega\right]^{-p^{\prime} q} .
$$

We are left with (3.7). Defining now

$$
k_{j}:=\mu^{+}-2^{-j} \widetilde{\omega},
$$

$j \leq \bar{\jmath}$, where $2^{-(\bar{\jmath}+2)}<2 \varepsilon_{2} \leq 2^{-(\bar{\jmath}+1)}$, we notice that the proof, which on the other hand follows closely that of (3.9), reduces to the proof for the standard evolutionary $p$-Laplacian, because the phase transition lies outside of the image of $w_{j}$ : indeed $b+\varepsilon \leq \mu^{+}-2 \widetilde{\omega}+\frac{\widetilde{\omega}}{2}<k_{j}$ for $j \in \mathbb{N}_{0}$, as a consequence of (Alt.1) and (3.6) 2 . Hence the singular term drops from the Caccioppoli's inequality and the time scale $T^{2}$ rebalances it as in the usual case: for details see, for example, $[6,8,15]$ and the forthcoming (3.21).

3.1.2. The geometric setting. Due to the three different cases we consider (and subsequently, with the three different time scales needed), we shall need to work with three families of shrinking cylinders and related cutoff functions.

Set, for $j \in \mathbb{N}_{0}$,

$$
\sigma_{j}:=\frac{1}{16}\left(1+2^{-j}\right), \quad \widetilde{\sigma}_{j}:=\frac{1}{4}\left(1+2^{-j}\right),
$$

and

$$
\begin{gathered}
Q_{j}^{i}:=\sigma_{j} Q^{i}=\left(B_{j} \times\left(t_{0}-T_{j}^{i}, t_{0}\right)\right) \cap \Omega_{T}, \quad i=1,2, \\
Q_{j}^{3}:=\widetilde{\sigma}_{j} Q^{3}=\left(\widetilde{B}_{j} \times\left(t_{0}-T_{j}^{3}, t_{0}\right)\right) \cap \Omega_{T},
\end{gathered}
$$

where

$$
B_{j}:=B_{\sigma_{j} r}\left(x_{0}\right), \quad T_{j}^{i}:=\sigma_{j} T^{i}, \quad i=1,2, \quad \widetilde{B}_{j}:=B_{\widetilde{\sigma}_{j} r}\left(x_{0}\right), \quad T_{j}^{3}:=\widetilde{\sigma}_{j} T^{3} .
$$

Note that

$$
\frac{1}{8} Q^{1}=Q_{0}^{1} \supset Q_{j}^{1} \stackrel{j \rightarrow \infty}{\longrightarrow} \frac{1}{16} Q^{1}, \quad \frac{1}{8} Q^{2}=Q_{0}^{2} \supset Q_{j}^{2} \stackrel{j \rightarrow \infty}{\longrightarrow} \frac{1}{16} Q^{2},
$$

and

$$
\frac{1}{2} Q^{3}=Q_{0}^{3} \supset Q_{j}^{3} \stackrel{j \rightarrow \infty}{\longrightarrow} \frac{1}{4} Q^{3} .
$$

We will take, for $i=1,2,3$ and $j \in \mathbb{N}_{0}$, standard smooth cut-off functions $\phi_{i, j}$ such that $\phi_{i, j}$ vanishes on the parabolic boundary of $Q_{j}^{i}$; moreover we assume $0 \leq \phi_{i, j} \leq 1$ and $\phi_{i, j} \equiv 1$ on $Q_{j+1}^{i}$. Note that we may also require

$$
\left|\partial_{t} \phi_{i, j}^{p}\right| \leq c(p) \frac{2^{j}}{T^{i}}, \quad\left|D \phi_{i, j}\right| \leq c \frac{2^{j}}{r} .
$$

Copyright $@$ by SIAM. Unauthorized reproduction of this article is prohibited. 
3.1.3. Occurrence of (Alt. 1). Here we state that using (3.7) it is possible to show that

$$
\sup _{\frac{1}{16} Q^{2}} v \leq \mu^{+}-\varepsilon_{2} \widetilde{\omega},
$$

provided we choose $\varepsilon_{2} \equiv \varepsilon_{2}(n, p, \Lambda, \delta)$ small enough. Indeed, the proof for the above fact reduces (more or less) to the analysis of the standard evolutionary $p$-Laplacian operator, because the phase transition lies outside of the support of the test functions; essentially, we follow the proof of [6, Chapter III, Lemma 9.1], once having (3.7) at hand. We sketch the proof for the convenience of the reader.

Choose for $j \in \mathbb{N}_{0}$ the levels

$$
k_{j}:=\mu^{+}-\left(1+2^{-j}\right) \varepsilon_{2} \widetilde{\omega},
$$

and consider the Caccioppoli inequality, Lemma (3.1), with $Q=Q_{j}^{2}, k=k_{j}$, and $\phi=\phi_{2, j}$. Noting that $k_{j} \geq \mu^{+}-\frac{3}{2} \widetilde{\omega} \geq b+\varepsilon$ and recalling that $T^{2}=\left[\varepsilon_{2} \widetilde{\omega}\right]^{2-p} r^{p}$ we have

$$
\begin{aligned}
& \frac{1}{\min \left\{T_{j}^{2}, t_{0}\right\}} \sup _{\max \left\{0, t_{0}-T_{j}^{2}\right\}<t<t_{0}} f_{B_{j} \cap \Omega}\left[\left(v-k_{j}\right)_{+}^{2} \phi_{2, j}^{p}\right](\cdot, t) d x \\
& +f_{Q_{j}^{2}}\left|D\left(v-k_{j}\right)_{+} \phi_{2, j}\right|^{p} d x d t \\
& \quad \leq c \frac{2^{j p}}{r^{p}} f_{Q_{j}^{2}}\left(\left(v-k_{j}\right)_{+}^{p}+\left(v-k_{j}\right)_{+}^{2}\left[\varepsilon_{2} \widetilde{\omega}\right]^{p-2}\right) d x d t
\end{aligned}
$$

with $c \equiv c(n, p, \Lambda)$. Using

$$
2^{-(j+1)} \varepsilon_{2} \widetilde{\omega} \chi_{\left\{v>k_{j+1}\right\}} \leq\left(v-k_{j}\right)_{+} \leq 2 \varepsilon_{2} \widetilde{\omega}
$$

and Sobolev's inequality (2.10) (see also Remark 3.2) together with (3.21), we have for all $j \in \mathbb{N}_{0}$

$$
\begin{aligned}
& {\left[2^{-(j+1)} \varepsilon_{2} \widetilde{\omega}\right]^{2(1-1 / \kappa)+p} A_{j+1} \leq f_{Q_{j+1}^{2}}\left(v-k_{j}\right)_{+}^{2(1-1 / \kappa)+p} d x d t} \\
& \begin{array}{c}
\leq r^{p}\left[T^{2}\right]^{1-1 / \kappa}\left[\frac{1}{\min \left\{T_{j}^{2}, t_{0}\right\}} \sup _{\max \left\{0, t_{0}-T_{j}^{2}\right\}<t<t_{0}} f_{B_{j} \cap \Omega}\left[\left(v-k_{j}\right)_{+}^{2} \phi_{2, j}^{p}\right](\cdot, t) d x\right]^{1-1 / \kappa} \\
\quad \times f_{Q_{j}^{2}}\left|D\left(v-k_{j}\right)_{+} \phi_{2, j}\right|^{p} d x d t
\end{array} \\
& \leq c r^{p}\left[T^{2}\right]^{1-1 / \kappa}\left[\frac{2^{j p}}{r^{p}} f_{Q_{j}^{2}}\left(\left(v-k_{j}\right)_{+}^{p}+\left(v-k_{j}\right)_{+}^{2}\left[\varepsilon_{2} \widetilde{\omega}\right]^{p-2}\right) d x d t\right]^{2-1 / \kappa} \\
& \leq c 2^{(2-1 / \kappa) p j} r^{p+p(1-1 / \kappa)-p(2-1 / \kappa)}\left[\varepsilon_{2} \widetilde{\omega}\right]^{(1-1 / \kappa)(2-p)+p(2-1 / \kappa)} \bar{A}_{j}^{2-1 / \kappa}
\end{aligned}
$$

with

$$
A_{j}:=f_{Q_{j}^{2}} \chi_{\left\{v>k_{j}\right\}} d x d t=\frac{\left|Q_{j}^{2} \cap\left\{v>k_{j}\right\}\right|}{\left|Q_{j}^{2}\right|} .
$$

Thus $A_{j+1} \leq c 2^{c(p, \kappa) j} A_{j}^{2-1 / \kappa}$, with $c$ depending on $n, p, \Lambda, 1 / \kappa$. This yields (3.20) in view of (3.7) and a standard hypergeometric iteration lemma, provided $\varepsilon_{2}$ is chosen small enough, in dependence of $n, p, \Lambda, \delta$, and $q$. Recall that $\kappa \equiv \kappa(q)$.

Copyright $@$ by SIAM. Unauthorized reproduction of this article is prohibited. 
3.1.4. Occurrence of (Alt.2) and (Alt. 2.1). Set for $j \in \mathbb{N}_{0}$

$$
k_{j}:=\mu^{+}-4\left(1+2^{-j}\right) \widetilde{\omega}
$$

and notice that $k_{j}<\mu^{+}-4 \widetilde{\omega}$, which together with (Alt. 2) and (3.6) ${ }_{2}$ implies $b-\varepsilon-$ $k_{j} \geq \widetilde{\omega}$. Thus, using (Alt. 2.1) we obtain

$$
\begin{aligned}
\sup _{\max \left\{0, t_{0}-T_{j+1}^{3}\right\}<t<t_{0}} & f_{\widetilde{B}_{j+1} \cap \Omega} \int_{k_{j}}^{v(\cdot, t)} H_{b, \varepsilon}^{\prime}(\xi)\left(\xi-k_{j}\right)_{+} d \xi d x \\
& \geq \sup _{\max \left\{0, t_{0}-\frac{1}{4} T^{3}\right\}<t<t_{0}} f_{B_{r / 4} \cap \Omega} \int_{b-\varepsilon}^{v(\cdot, t)} H_{b, \varepsilon}^{\prime}(\xi)\left(\xi-k_{j}\right)_{+} d \xi d x \\
& \geq \widetilde{\omega} \sup _{\max \left\{0, t_{0}-\frac{1}{4} T^{1}\right\}<t<t_{0}} f_{B_{r / 4} \cap \Omega} \int_{\mu^{+}-3 \widetilde{\omega}}^{v(\cdot, t)} H_{b, \varepsilon}^{\prime}(\xi) d \xi d x \\
& >\varepsilon_{3}^{-1} \widetilde{\omega}\left[\varepsilon_{1} \omega\right]^{q},
\end{aligned}
$$

in view of (3.4). By Poincaré's inequality (see Remark 3.2) we have

$$
f_{Q_{j+1}^{3}}\left(v-k_{j}\right)_{+}^{p} d x d t \leq c(n, p, \delta) r^{p} f_{Q_{j+1}^{3}}\left|D\left(v-k_{j}\right)_{+}\right|^{p} d x d t,
$$

which together with (3.23) and the Caccioppoli inequality with $Q=Q_{j}^{3}, k=k_{j}$, and $\phi=\phi_{3, j}$ yields

$$
\begin{aligned}
& \frac{\varepsilon_{3}^{-1} \widetilde{\omega}\left[\varepsilon_{1} \omega\right]^{q}}{T^{3}} f_{Q_{j+1}^{3}}\left(v-k_{j}\right)_{+}^{p} d x d t \\
& \leq c\left[\frac{1}{\min \left\{T_{j+1}^{3}, t_{0}\right\}} \sup _{\max \left\{0, t_{0}-T_{j+1}^{3}\right\}<t<t_{0}} f_{\widetilde{B}_{j+1} \cap \Omega} \int_{k_{j}}^{v(\cdot, t)} H_{b, \varepsilon}^{\prime}(\xi)\left(\xi-k_{j}\right)_{+} d \xi d x\right] \\
& \times\left[r^{p} f_{Q_{j+1}^{3}}\left|D\left(v-k_{j}\right)_{+}\right|^{p} d x d t\right] \\
& \leq c 2^{2 p j} r^{p}\left[f_{Q_{j}^{3}}\left(\frac{\left(v-k_{j}\right)_{+}^{p}}{r^{p}}+\frac{\left(v-k_{j}\right)_{+}^{2}}{T^{3}}\right) d x d t\right. \\
& \left.+f_{Q_{j}^{3}} \int_{k_{j}}^{v} H_{b, \varepsilon}^{\prime}(\xi) \frac{\left(\xi-k_{j}\right)_{+}}{T^{3}} d \xi d x d t\right]^{2} .
\end{aligned}
$$

At this point, to bound both the left- and the right-hand side, we use the following facts: first, we have

$$
2^{-(j-1)} \widetilde{\omega} \chi_{\left\{v>k_{j+1}\right\}} \leq\left(v-k_{j}\right)_{+} \leq 8 \widetilde{\omega} \chi_{\left\{v>k_{j}\right\}} ;
$$

then, the definition of $T^{3}=\widetilde{\omega}^{1-p} r^{p}$ and also the fact that $\widetilde{\omega} \leq 1$ yield

$$
2^{-p j} \varepsilon_{3}^{-1}\left[\varepsilon_{1} \omega\right]^{q} f_{Q_{j+1}^{3}} \chi_{\left\{v>k_{j+1}\right\}} d x d t \leq c 2^{2 p j}\left(f_{Q_{j}^{3}} \chi_{\left\{v>k_{j}\right\}} d x d t\right)^{2}
$$

with $c \equiv c(n, p, \Lambda, \delta)$. Denoting

$$
\bar{A}_{j}:=f_{Q_{j}^{3}} \chi_{\left\{v>k_{j}\right\}} d x d t=\frac{\left|Q_{j}^{3} \cap\left\{v>k_{j}\right\}\right|}{\left|Q_{j}^{3}\right|}
$$

Copyright $@$ by SIAM. Unauthorized reproduction of this article is prohibited. 
we hence finally have

$$
\bar{A}_{j+1} \leq 2^{3 p j} \bar{c} \varepsilon_{3}\left[\varepsilon_{1} \omega\right]^{-q} \bar{A}_{j}^{2},
$$

where the constant $\bar{c}$ depends on $n, p, \Lambda, \delta$, but it is independent of $\varepsilon_{1}$. Then, if

$$
\bar{A}_{0} \leq \frac{\left[\varepsilon_{1} \omega\right]^{q}}{\varepsilon_{3} 2^{3 p} \bar{c}}
$$

the sequence $\left\{A_{j}\right\}$ becomes infinitesimal, in particular implying that

$$
\sup _{\frac{1}{4} Q^{3}} v \leq \mu^{+}-4 \widetilde{\omega} .
$$

The above condition for $\bar{A}_{0}$ can be certainly guaranteed by taking

$$
\varepsilon_{3}:=\frac{1}{2^{3 p} c_{\ell} \bar{c}}
$$

since Lemma 3.3, (3.8), gives us exactly

$$
\frac{\left|Q_{0}^{3} \cap\left\{v>k_{0}\right\}\right|}{\left|Q_{0}^{3}\right|} \leq c_{\ell}\left[\varepsilon_{1} \omega\right]^{q} ;
$$

recall that we are assuming here (Alt. 2). Note carefully that now the parameter $\varepsilon_{3}$ has been fixed as a parameter of $n, p, \Lambda, \delta$, but it is independent of $\varepsilon_{1}$.

3.1.5. Occurrence of (Alt.2) and (Alt. 2.2). We set this time for $j \in \mathbb{N}_{0}$,

$$
k_{j}:=\mu^{+}-\left(1+2^{-j}\right) \varepsilon_{1} \omega .
$$

Choosing $Q=Q_{j}^{1}, k=k_{j}$, and $\phi=\phi_{1, j}$ the Caccioppoli's estimate takes the form

$$
\begin{aligned}
\frac{1}{\min \left\{T_{j}^{1}, t_{0}\right\}} \sup _{\max \left\{0, t_{0}-T_{j}^{1}\right\}<t<t_{0}} f_{B_{j} \cap \Omega}\left(\left(v-k_{j}\right)_{+}^{2} \phi_{1, j}^{p}\right)(\cdot, t) d x & \\
& +f_{Q_{j}^{1}}\left|D\left(v-k_{j}\right)_{+} \phi_{1, j}\right|^{p} d x d t \\
\leq c 2^{p j}\left[f_{Q_{j}^{1}}\left(\frac{\left(v-k_{j}\right)_{+}^{p}}{r^{p}}+\frac{\left(v-k_{j}\right)_{+}^{2}}{T^{1}}\right) d x d t\right. & \\
& \left.+\frac{1}{T^{1}} f_{Q_{j}^{1}} \int_{k_{j}}^{v} H_{b, \varepsilon}^{\prime}(\xi)\left(\xi-k_{j}\right)_{+} d \xi d x d t\right] .
\end{aligned}
$$

Now using $\left(v-k_{j}\right)_{+} \leq 2 \varepsilon_{1} \omega$, Hölder's inequality, and (Alt. 2.2) yields

$$
\begin{aligned}
& f_{Q_{j}^{1}} \int_{k_{j}}^{v} H_{b, \varepsilon}^{\prime}(\xi)\left(\xi-k_{j}\right)_{+} d \xi d x d t \\
& \leq 2 \varepsilon_{1} \omega\left(f_{Q_{j}^{1}}\left[\int_{\mu^{+}-3 \widetilde{\omega}}^{v} H_{b, \varepsilon}^{\prime}(\xi) d \xi\right]^{q} d x d t\right)^{\frac{1}{q}}\left(f_{Q_{j}^{1}} \chi_{\left\{v>k_{j}\right\}} d x d t\right)^{1-\frac{1}{q}} \\
& \leq 2 \varepsilon_{1} \omega\left(\sup _{\max \left\{0, t_{0}-T_{j}^{1}\right\}<t<t_{0}} f_{B_{r / 4} \cap \Omega} \int_{\mu^{+}-3 \widetilde{\omega}}^{v(\cdot, t)} H_{b, \varepsilon}^{\prime}(\xi) d \xi d x\right)^{\frac{1}{q}}\left(f_{Q_{j}^{1}} \chi_{\left\{v>k_{j}\right\}} d x d t\right)^{1-\frac{1}{q}} \\
& \leq c \varepsilon_{3}^{-1 / q}\left[\varepsilon_{1} \omega\right]^{2} \widetilde{A}_{j}^{1-\frac{1}{q}},
\end{aligned}
$$


setting

$$
\widetilde{A}_{j}:=f_{Q_{j}^{1}} \chi_{\left\{v>k_{j}\right\}} d x d t=\frac{\left|Q_{j}^{1} \cap\left\{v>k_{j}\right\}\right|}{\left|Q_{j}^{1}\right|},
$$

recalling that $q>1$ and $\int_{\mathbb{R}} H_{b, \varepsilon}^{\prime} d \xi \leq 1$. Also recall that $\varepsilon_{3}$ is fixed and depends only on $n, p, \Lambda, \delta$. Combining the two displays above and recalling that $T^{1}=\left[\varepsilon_{1} \omega\right]^{2-p} r^{p}$, we obtain

$$
\begin{aligned}
\frac{1}{\min \left\{T_{j}^{1}, t_{0}\right\}} \sup _{\max \left\{0, t_{0}-T_{j}^{1}\right\}<t<t_{0}} f_{B_{j} \cap \Omega}\left(\left(v-k_{j}\right)_{+}^{2} \phi_{1, j}^{p}\right)(\cdot, t) d x \\
\quad+f_{Q_{j}^{1}}\left|D\left(v-k_{j}\right)_{+} \phi_{1, j}\right|^{p} d x d t \\
\leq c 2^{p j} \frac{\left[\varepsilon_{1} \omega\right]^{p}}{r^{p}}\left(\widetilde{A}_{j}+\varepsilon_{3}^{-1 / q} \widetilde{A}_{j}^{1-\frac{1}{q}}\right) \leq c \varepsilon_{3}^{-1 / q} 2^{p j} \frac{\left[\varepsilon_{1} \omega\right]^{p}}{r^{p}} \widetilde{A}_{j}^{1-1 / q} .
\end{aligned}
$$

To conclude, by Sobolev's inequality (2.10) with $\phi:=\phi_{1, j}, w=\left(v-k_{j}\right)_{+}, B=B_{j} \cap \Omega$, and $\Gamma=\left(\max \left\{0, t_{0}-T_{j}^{1}\right\}, t_{0}\right)$ we infer

$$
\begin{aligned}
& f_{Q_{j+1}^{1}}\left(v-k_{j}\right)_{+}^{2(1-1 / \kappa)+p} d x d t \\
& \leq c\left[\frac{1}{\min \left\{T_{j}^{1}, t_{0}\right\}} \sup _{\max \left\{0, t_{0}-T_{j}^{1}\right\}<t<t_{0}} f_{B_{j} \cap \Omega}\left(\left(v-k_{j}\right)_{+}^{2} \phi_{1, j}^{p}\right)(\cdot, t) d x\right]^{1-1 / \kappa} \\
& \times r^{p}\left[T^{1}\right]^{1-1 / \kappa} f_{Q_{j}^{1}}\left|D\left(v-k_{j}\right)_{+} \phi_{1, j}\right|^{p} d x d t \\
& \leq c \varepsilon_{3}^{-(2-1 / \kappa) / q} r^{p+p(1-1 / \kappa)-p(2-1 / \kappa)}\left[\varepsilon_{1} \omega\right]^{(2-p)(1-1 / \kappa)+p(2-1 / \kappa)} \\
& \times 2^{p(2-1 / \kappa) j} \widetilde{A}_{j}^{(1-1 / q)(2-1 / \kappa)},
\end{aligned}
$$

with $c$ depending on $n, p, \Lambda, q, \delta$. Estimating finally

$$
\left(v-k_{j}\right)_{+} \geq 2^{-(j+1)}\left[\varepsilon_{1} \omega\right] \chi_{\left\{v>k_{j+1}\right\}}
$$

we conclude with

$$
\widetilde{A}_{j+1} \leq \tilde{c} \varepsilon_{3}^{-(2-1 / \kappa) / q} 2^{4 p j} \widetilde{A}_{j}^{1+\zeta},
$$

where $\zeta:=(1-1 / q)(2-1 / \kappa)-1>0$ by $(2.12)$ and $\tilde{c}$ depends only on $n, p, \Lambda, \delta$, and $q$. Hence by choosing

$$
\varepsilon_{1}:=\min \left\{\exp \left[-\left(c_{\ell} \tilde{c}^{1 / \zeta} 2^{4 p / \zeta^{2}} \varepsilon_{3}^{-(1 / p+(2-1 / \kappa) /(\zeta q))}\right)^{p^{\prime}}\right], \varepsilon_{2}^{p-2}, \varepsilon_{2}, 2^{-10}\right\}
$$

we get by (3.9) that

$$
\widetilde{A}_{0} \leq\left[\tilde{c} \varepsilon_{3}^{-(2-1 / \kappa) / q}\right]^{-1 / \zeta} 2^{-4 p / \zeta^{2}}
$$

and again a standard hypergeometric iteration lemma ensures that

$$
\sup _{\frac{1}{16} Q^{1}} v \leq \mu^{+}-\varepsilon_{1} \omega .
$$

Note that, taking into account the fact that $\varepsilon_{2}$ has already been fixed in subsection 3.1.3 as constant depending on $n, p, \Lambda, \delta$, and $q$ and also $\varepsilon_{3}$ has been fixed in (3.26) depending only on $n, p, \Lambda, \delta$, now also $\varepsilon_{1}$ is fixed as a constant depending only on $n, p, \Lambda, \delta$, and $q$. 
3.1.6. Conclusion. All in all, merging the three different alternatives that yield (3.20), (3.25), and (3.28), then adding $-\inf _{\frac{1}{16} Q^{1}} v \leq-\mu^{-}$, we have proved that if $v$ is a solution to (3.1) and (3.6) holds, then

$$
\underset{\frac{1}{16} Q^{1}}{\operatorname{osc}} v \leq \underset{Q^{3}}{\operatorname{osc}} v-\varepsilon_{1} \widetilde{\omega} \leq \underset{Q^{3}}{\operatorname{osc}} v-\varepsilon_{1}^{2} \omega \exp \left(-\left[\varepsilon_{1} \omega\right]^{-p^{\prime} q}\right) .
$$

Indeed if $b$ satisfies (3.5), then (3.29) is what we proved on the previous pages. On the other hand, if $b \notin\left[\inf _{Q^{3}} v, \sup _{Q^{3}} v\right]$, we are essentially in the same situation as described in subsection 3.1.3 and therefore also in this case (3.20), and hence (3.29), holds. Note that if $b \notin\left[\inf _{Q^{3}} v, \sup _{Q^{3}} v\right]$, then for $\varepsilon$ small enough $v$ is a solution to the evolutionary $p$-Laplace equation, and the oscillation reduction follows in general by the well-known argument of DiBenedetto (see $[6,15]$ ); however, referring also in this case to subsection 3.1.3 allows for a unitary treatment of these alternatives.

Remark 3.4. Note that in case

$$
\inf _{\bar{Q}^{3} \cap \partial_{p} \Omega_{T}} \tilde{g} \geq \mu^{-}+\frac{\omega}{8}, \quad \varepsilon \leq \frac{\widetilde{\omega}}{2},
$$

holds in place of (3.6), then (3.29) still holds since $-v$ solves an equation similar to (3.1) with boundary datum $-\tilde{g}$.

3.2. Reducing the oscillation at the initial boundary. Let us take $x_{0} \in \bar{\Omega}$. Similarly to the previous subsection, here we denote, for some $\omega>0$,

$$
Q:=\left(B_{r}\left(x_{0}\right) \cap \Omega\right) \times\left(0, T^{4}\right), \quad T^{4}:=\min \left\{\omega^{2-p} r^{p}, T\right\}
$$

and we consider the function $v$ solving (3.1) with Cauchy-Dirichlet datum $\tilde{g}$. Let us remind the reader that the Caccioppoli's inequality of Lemma 3.1 is valid for $v$ also in this case. We can then follow the steps in [6, Chapter III, section 11] using time independent cut-off functions and we can reduce the problem to the analysis of the standard evolutionary $p$-Laplace equation; we briefly present the proof adapted to our setting.

The next result is a standard "logarithmic lemma"; see, for example, the proof in $[6$, Chapter II]. The assumption in (3.32) will be satisfied by imposing a proper condition between the solution and the initial trace $g(\cdot, 0)$; see $(4.7)$.

Lemma 3.5. Let $Q$ and $T^{4}$ be as in (3.31), and assume that $v \in C(\bar{Q})$ solves (3.1) in $Q$ and

$$
\sup _{B_{r}\left(x_{0}\right) \cap \Omega} v(\cdot, 0) \leq \sup _{Q} v-\frac{\omega}{8} .
$$

Then, for a constant $c$ depending on $n, p, \Lambda$, there holds

$$
\frac{\left|\left(B_{r / 2}\left(x_{0}\right) \cap \Omega\right) \cap\left\{v(\cdot, \tau) \geq \sup _{Q} v-\theta \omega / 8\right\}\right|}{\left|B_{r / 2}\left(x_{0}\right) \cap \Omega\right|} \leq \frac{c}{\log (1 / \theta)}
$$

whenever $\theta \in(0,1)$ and $\tau \in\left(0, T^{4}\right)$.

Proof. Denote in short $\widetilde{\mathcal{A}}(D v):=\widetilde{\mathcal{A}}(x, t, v, D v), \hat{B}:=B_{r}\left(x_{0}\right) \cap \Omega$, and $\mathcal{H}$ as in (2.7), with $b$ replacing $a$. Consider a time independent cut-off function $\phi \in$ 
$C_{c}^{\infty}\left(B_{r}\left(x_{0}\right)\right), 0 \leq \phi \leq 1$, with $\phi \equiv 1$ in $B_{r / 2}, \phi=0$ on $\partial B_{r}\left(x_{0}\right)$, and $|D \phi| \leq c / r$. Take $k=\sup _{Q} v-\omega / 8$ and define for $\theta \in(0,1 / 8]$ the function

$$
\Psi(v)=\left[\log \left(\frac{\omega}{\omega(1+\theta)-8(v-k)_{+}}\right)\right]_{+} .
$$

We have $\Psi(v) \neq 0$ when $v>\sup _{Q} v-\omega(1-\theta) / 8=: v_{-}>\sup _{Q} v-\omega / 8>\omega / 2$ (note that if $\sup _{Q} v \leq 3 \omega / 4$ there is nothing to prove, since (3.33) would be trivial). Observe that we have

$$
\Psi^{\prime}(v)=\chi_{\left\{v>v_{-}\right\}} \frac{8}{\omega(1+\theta)-8(v-k)_{+}} .
$$

Testing formally the equation with $\eta=\Psi^{\prime}(v) \Psi(v) \phi^{p} \chi_{(-\infty, \tau)}(t)$, for $\tau \in\left(0, T^{4}\right)$, which vanishes in a neighborhood of $\partial_{p} \Omega_{T}$ being continuous and zero on $\partial_{p} \Omega_{T}$, we have

$$
-\int_{\hat{B} \times(0, \tau)}\langle\widetilde{\mathcal{A}}(D v), D \eta\rangle d x d t=\int_{\hat{B} \times(0, \tau)} \partial_{t} \mathcal{H}(v) \eta d x d t .
$$

To be precise, this choice of the test function is admissible only after a suitable mollification in time; see, for instance, the steps in the end of the proof of [1, Lemma 2.3] for a rigorous treatment of the parabolic term in this setting. Indeed one should prove the estimate not directly up to $t=0$ but $t=\varepsilon$, for $\varepsilon$ (the mollification parameter) small enough, and then pass to the limit. We have

$$
\partial_{t} \mathcal{H}(v) \Psi^{\prime}(v) \Psi(v)=\partial_{t} \int_{v_{-}}^{v} \mathcal{H}^{\prime}(\xi) \Psi^{\prime}(\xi) \Psi(\xi) d \xi
$$

and integration by parts gives

$$
\int_{\hat{B} \times(0, \tau)} \partial_{t} \mathcal{H}(v) \Psi^{\prime}(v) \Psi(v) \phi^{p} d x d t=\left.\int_{\hat{B}} \int_{v_{-}}^{v(\cdot, t)} \mathcal{H}^{\prime}(\xi) \Psi^{\prime}(\xi) \Psi(\xi) d \xi \phi^{p} d x\right|_{t=0} ^{\tau},
$$

since $\phi$ is time independent and recalling that $v \in C(\bar{Q})$. Since $v<v_{-}$on $\hat{B} \times\{0\}$, we have that the term on the right-hand side for $t=0$ is zero. Therefore

$$
\int_{\hat{B} \times(0, \tau)} \partial_{t} \mathcal{H}(v) \Psi^{\prime}(v) \Psi(v) \phi^{p} d x d t=\int_{\hat{B}} \int_{v_{-}}^{v(x, \tau)} \mathcal{H}^{\prime}(\xi) \Psi^{\prime}(\xi) \Psi(\xi) d \xi \phi(x)^{p} d x
$$

and since $\mathcal{H}^{\prime} \geq 1$ and $\Psi\left(v_{-}\right)=0$, we obtain

$$
\int_{\hat{B}} \Psi^{2}(v(\cdot, \tau)) \phi^{p} d x \leq 2 \int_{\hat{B} \times(0, \tau)} \partial_{t} \mathcal{H}(v) \Psi^{\prime}(v) \Psi(v) \phi^{p} d x d t .
$$

As for the elliptic term, we get from (2.6)

$$
\begin{aligned}
-\int_{\hat{B} \times(0, \tau)}\langle\widetilde{\mathcal{A}}(D v), D \eta\rangle d x d t=- & \int_{\hat{B} \times(0, \tau)}\langle\widetilde{\mathcal{A}}(D v), D v\rangle(1+\Psi(v))\left[\Psi^{\prime}(v)\right]^{2} \phi^{p} d x d t \\
& -\int_{\hat{B} \times(0, \tau)}\left\langle\widetilde{\mathcal{A}}(D v), D \phi^{p}\right\rangle \Psi^{\prime}(v) \Psi(v) d x d t
\end{aligned}
$$

Copyright $@$ by SIAM. Unauthorized reproduction of this article is prohibited. 


$$
\begin{aligned}
\leq- & (p, \Lambda) \int_{\hat{B} \times(0, \tau)}|D v|^{p}(1+\Psi(v))\left[\Psi^{\prime}(v)\right]^{2} \phi^{p} d x d t \\
& +c(p, \Lambda) \int_{Q} \Psi(v)\left[\Psi^{\prime}(v)\right]^{2-p}|D \phi|^{p} d x d t,
\end{aligned}
$$

using Young's inequality. We thus obtain, discarding the negative term on the righthand side,

$$
\int_{\hat{B}} \Psi^{2}(v(\cdot, \tau)) \phi^{p} d x \leq c \int_{Q} \Psi(v)\left[\Psi^{\prime}(v)\right]^{2-p}|D \phi|^{p} d x d t
$$

this holds for all $\tau \in\left(0, T^{4}\right]$. The very definitions of $\Psi$ and $T^{4}$ then imply

$$
\int_{\frac{1}{2} \hat{B}}[\Psi(v(\cdot, \tau))]^{2} d x \leq c \frac{|\hat{B}| T^{4}}{r^{p}} \log \frac{1}{\theta}\left(\frac{\omega}{8}\right)^{p-2} \leq c\left|\frac{1}{2} \hat{B}\right| \log \frac{1}{\theta},
$$

since $\theta \omega / 8<(v-k)_{+} \leq \omega / 8$ in $\{\Psi(v) \neq 0\}$. Moreover, the left-hand side can be bounded from below as

$$
\int_{\frac{1}{2} \hat{B}}[\Psi(v(\cdot, \tau))]^{2} d x \geq\left|\frac{1}{2} \hat{B} \cap\left\{v(\cdot, \tau) \geq \sup _{Q} v-\theta \omega / 8\right\}\right|\left(\log \frac{1}{2 \theta}\right)^{2}
$$

and we conclude with

$$
\frac{\left|\frac{1}{2} \hat{B} \cap\left\{v(\cdot, \tau) \geq \sup _{Q} v-\theta \omega / 8\right\}\right|}{\left|\frac{1}{2} \hat{B}\right|} \leq c \frac{\log (1 / \theta)}{[\log (1 /(2 \theta))]^{2}} \leq \frac{c}{\log (1 / \theta)} .
$$

Therefore, if (3.32) holds, then for all $\nu_{*} \in(0,1)$ we find $\varepsilon_{4} \equiv \varepsilon_{4}\left(n, p, \Lambda, \nu_{*}\right)$ such that after integration, denoting $\sigma Q:=\left(B_{\sigma r} \cap \Omega\right) \times(0, T)$ for $\sigma \in(0,1]$, we have

$$
\left|\frac{1}{2} Q \cap\left\{v \geq \sup _{Q} v-2 \varepsilon_{4} \omega\right\}\right| \leq \nu_{*}\left|\frac{1}{2} Q\right| .
$$

We can now deduce the following.

Proposition 3.6. Let $v$ be a solution to (3.1) in $Q$ and suppose that (3.32) holds for some $\omega>0$. Then

$$
\sup _{\frac{1}{4} Q} v \leq \sup _{Q} v-\varepsilon_{4} \omega,
$$

where $\varepsilon_{4}$ is a constant depending on $n, p, \Lambda, \delta$, and $q$.

Proof. Note that taking independent of time cut-off functions, the Caccioppoli's inequality does not contain the terms containing $H_{b, \varepsilon}^{\prime}$ on the right-hand side. In particular we set

$$
Q_{j}:=\left(B_{\sigma_{j} r}\left(x_{0}\right) \cap \Omega\right) \times\left(0, T^{4}\right)=: B_{j} \times\left(0, T^{4}\right), \quad \sigma_{j}=\frac{1}{4}\left(1+2^{-j}\right),
$$

and we have

$$
\begin{aligned}
\frac{1}{T^{4}} \sup _{0<t<T^{4}} f_{B_{j+1}}\left[(v-k)_{+}^{2}\right](\cdot, t) d x+f_{Q_{j+1}}\left|D(v-k)_{+}\right|^{p} d x d t & \\
& \leq c 2^{j p} f_{Q_{j}} \frac{(v-k)_{+}^{p}}{r^{p}} d x d t .
\end{aligned}
$$

Copyright $@$ ㅇ by SIAM. Unauthorized reproduction of this article is prohibited. 
Setting $k_{j}:=\sup _{Q} v-\left(1+2^{-j}\right) \varepsilon_{4} \omega$ and using Sobolev's inequality (2.10) (possibly the boundary version mentioned in the last remark of subsection 2.4) we infer, with $\kappa$ defined in (2.9) and the agreement in (2.11),

$$
\begin{aligned}
& f_{Q_{j+1}}\left(v-k_{j}\right)_{+}^{2(1-1 / \kappa)+p} d x d t \\
& \quad \leq c(n, p, \delta) r^{p}\left[T^{4}\right]^{1-1 / \kappa}\left[\frac{2^{j p}}{r^{p}} f_{Q_{j}}\left(v-k_{j}\right)_{+}^{p} d x d t\right]^{2-1 / \kappa} \\
& \quad \leq c 2^{c(p, \kappa) j} \omega^{(1-1 / \kappa)(2-p)+p(2-1 / \kappa)}\left[f_{Q_{j}} \chi_{\left\{v>k_{j}\right\}} d x d t\right]^{2-1 / \kappa} .
\end{aligned}
$$

Note all this is possible since $k_{j} \geq \sup _{Q} v-\omega / 8$ when $\varepsilon_{4}$ is small enough, and hence $\left(v-k_{j}\right)_{+}$vanishes in a neighborhood of $\partial_{\text {par }} \Omega_{T}$ by the boundary continuity of $v$. Now reasoning as after (3.22), a standard hypergeometric iteration lemma yields (3.34) provided that $\nu_{*}$ is chosen small enough, depending on $n, p, \Lambda, \delta$, and $q$; this finally fixes $\varepsilon_{4}$.

4. The approximate boundary continuity. The goal of this section is the iteration of the results of the previous section; this will give in a standard way, as a consequence, the boundary continuity. Moreover, we shall show how to explicitly infer the modulus described in (1.7).

4.1. Iterative estimates. The goal of the next proposition will be twofold. On the one hand, we show how to set the estimates (3.29) and (3.34) into an iterative scheme. On the other hand, we unify the interior (presented in [1]), initial, and lateral boundary cases in order to have estimates slightly more manageable.

Proposition 4.1. Let $R_{0} \leq r_{\Omega},\left(x_{0}, t_{0}\right) \in \bar{\Omega}_{T}$ and $q>\bar{q}$, where $\bar{q} \geq 2$ has been defined in (1.5); set

$$
\alpha:=\frac{1}{p^{\prime} q} \in\left(0, \frac{1}{p^{\prime} \bar{q}}\right) .
$$

Then there exist constants $\vartheta, \tau \in(0,1 / 2)$ depending only on $n, p, \Lambda, \delta$, and $q$ such that for any decreasing sequence $\left\{\omega_{j}\right\}_{j \in \mathbb{N}_{0}}$ with

$$
\omega_{0}:=1, \quad \omega_{j+1} \geq \omega_{j}\left(1-\vartheta \exp \left(-\left[\vartheta \omega_{j}\right]^{-1 / \alpha}\right)\right),
$$

and moreover defining for $j \in \mathbb{N}_{0}$

$$
\begin{aligned}
& \widetilde{\omega}_{j}:=\tau \omega_{j} \exp \left(-\left[\tau \omega_{j}\right]^{-1 / \alpha}\right), \\
& R_{j+1}:=\exp \left(-\frac{\vartheta}{\alpha}\left[\vartheta \omega_{j}\right]^{-1 / \alpha}\right) R_{j}, \quad T_{j}:=\widetilde{\omega}_{j}^{1-p} R_{j}^{p}, \\
& Q^{j}:=\left(B_{R_{j}}\left(x_{0}\right) \times\left(t_{0}-T_{j}, t_{0}+T_{j}\right)\right) \cap \bar{\Omega}_{T},
\end{aligned}
$$

we have the following: If $v$ is a continuous weak solution to (3.1) in $Q^{j}$ with $\varepsilon \leq \widetilde{\omega}_{j} / 2$ and such that

$$
\underset{Q^{j}}{\operatorname{Osc} v} \leq \omega_{j}
$$

for some $j \in \mathbb{N}_{0}$, then

$$
\underset{Q^{j+1}}{\operatorname{Osc}} v \leq \max \left\{\omega_{j+1}, 2 \underset{\bar{Q}^{j} \cap \partial_{p} \Omega_{T}}{\operatorname{osc}} \tilde{g}\right\} .
$$

Copyright $@$ by SIAM. Unauthorized reproduction of this article is prohibited. 
Proof. Fix $j \in \mathbb{N}_{0}$ as in the statement of the proposition and suppose that (4.3) holds. Observe that by considering the time $t_{j}:=t_{0}+T_{j}$ instead of $t_{0}$, we may write both $Q^{j}$ and $Q^{j+1}$ as backward in time cylinders:

$$
Q^{i}=\left(B_{R_{i}}\left(x_{0}\right) \times\left(t_{i}-2 T_{i}, t_{i}\right)\right) \cap \bar{\Omega}_{T}
$$

for $i=j, j+1$. Notice that it could indeed happen that $t_{j}, t_{j+1}>T$. In order to have some freedom we choose two auxiliary parameters

$$
\widetilde{R}_{j}:=\exp \left(-\frac{2 \vartheta}{3 \alpha}\left[\vartheta \omega_{j}\right]^{-1 / \alpha}\right) R_{j} \quad \text { and } \quad \hat{R}_{j}:=\exp \left(-\frac{\vartheta}{3 \alpha}\left[\vartheta \omega_{j}\right]^{-1 / \alpha}\right) R_{j} .
$$

Note that not only do we have $R_{j+1} \leq \widetilde{R}_{j} \leq \hat{R}_{j} \leq R_{j}$, but the ratios

$$
\frac{R_{j+1}}{\widetilde{R}_{j}}=\frac{\widetilde{R}_{j}}{\hat{R}_{j}}=\frac{\hat{R}_{j}}{R_{j}}=\exp \left(-\frac{\vartheta}{3 \alpha}\left[\vartheta \omega_{j}\right]^{-1 / \alpha}\right) \leq \exp \left(-\frac{\vartheta^{-1}}{3 \alpha}\right)
$$

can be made as small as we please by choosing $\vartheta$ small enough (note that $\alpha<1 / 4$ ). Moreover, we set

$$
Q_{\text {int }}(r, \omega):=B_{r}\left(x_{0}\right) \times\left(\bar{t}-\tilde{M} \omega^{(2-p)(1+1 / \tilde{\alpha})} r^{p}, \bar{t}\right), \quad \bar{t}:=\max \left\{t_{j+1}, T\right\} ;
$$

$\tilde{\alpha} \equiv \tilde{\alpha}(n, p)$ is the exponent appearing in [1, Theorem 1.2], relabeled; its explicit value is not important here, only the fact that $\tilde{\alpha} \in(0,1) . M$ is the constant appearing in [1, Theorem 1.2], larger than one and depending on $n, p, \Lambda$, and $\tilde{\alpha}$; note that the dependence on $\tilde{\alpha}$ is meaningful only in the case $p=n$. We fix, in this case, $\tilde{\alpha}=1 / 4$ so that in any case $\tilde{M}=\tilde{M}(n, p, \Lambda)$.

Case 1. Interior estimate. Let us first assume that $Q_{\text {int }}\left(\widetilde{R}_{j}, \omega_{j}\right) \subset \Omega_{T}$. Since $\widetilde{R}_{j} \leq R_{j}$ and

$$
\begin{aligned}
\frac{\tilde{M} \omega_{j}^{(2-p)(1+1 / \tilde{\alpha})} \widetilde{R}_{j}^{p}}{\widetilde{\omega}_{j}^{1-p} R_{j}^{p}} & =\tilde{M} \tau^{p-1} \omega_{j}^{1-(p-2) / \tilde{\alpha}} \exp \left(-(p-1)\left[\tau \omega_{j}\right]^{-1 / \alpha}\right)\left(\frac{\widetilde{R}_{j}}{R_{j}}\right)^{p} \\
& \leq \tilde{M} \tau^{(p-2)(1+1 / \tilde{\alpha})} \sup _{\varsigma \in(0,1)} \varsigma^{1-(p-2) / \tilde{\alpha}} \exp \left(-(p-1) \varsigma^{-1 / \alpha}\right) \\
& =: \tilde{M} \mathcal{S}(p, q) \tau^{(p-2)(1+1 / \tilde{\alpha})} \leq 1
\end{aligned}
$$

for small enough $\tau \equiv \tau(n, p, \Lambda, q)$, we have $Q_{\text {int }}\left(\widetilde{R}_{j}, \omega_{j}\right) \subset Q^{j}$. Using now [1, Remark $4.3]$ and the proof of $[1$, Theorem 4.1] we see that

$$
\underset{Q_{\text {int }}\left(\frac{1}{32} \widetilde{R}_{j}, \omega_{j}\right)}{\operatorname{OsC}} v \leq \omega_{j+1},
$$

and the inclusion $Q^{j+1} \subset Q_{\text {int }}\left(\frac{1}{32} \widetilde{R}_{j}, \omega_{j}\right)$ follows choosing small enough $\vartheta$ depending on $n, p, \Lambda, q$, and $\tau$. Indeed, first we take $\vartheta$ so that $e^{-\vartheta^{-1} /[3 \alpha]} \leq 1 / 32$ (see (4.5)). Then we notice that, since $\omega_{j+1} \geq \omega_{j} / 2$,

$$
\begin{aligned}
\frac{\widetilde{\omega}_{j+1}^{1-p} R_{j+1}^{p}}{\tilde{M} \omega_{j}^{(2-p)(1+1 / \tilde{\alpha})}\left(\widetilde{R}_{j} / 32\right)^{p}} & \leq c(p, \tilde{M})\left(\frac{\tau \omega_{j}}{2}\right)^{1-p} \frac{\exp \left((p-1)\left(\frac{\tau \omega_{j}}{2}\right)^{-1 / \alpha}\right)}{\omega_{j}^{(2-p)(1+1 / \tilde{\alpha})}}\left(\frac{R_{j+1}}{\widetilde{R}_{j}}\right)^{p} \\
& \leq \frac{c}{\tau^{p-1} \omega_{j}} \exp \left(\left[-\frac{p \vartheta^{1-1 / \alpha}}{3 \alpha}+(p-1)\left(\frac{\tau}{2}\right)^{-1 / \alpha}\right] \omega_{j}^{-1 / \alpha}\right)
\end{aligned}
$$

Copyright $@$ by SIAM. Unauthorized reproduction of this article is prohibited. 
Now if we choose $\vartheta \leq c(p, \alpha) \tau^{1 /(1-\alpha)}$ yielding

$$
\frac{\widetilde{\omega}_{j+1}^{1-p} R_{j+1}^{p}}{\tilde{M} \omega_{j}^{(2-p)(1+1 / \tilde{\alpha})}\left(\widetilde{R}_{j} / 32\right)^{p}} \leq \frac{c(n, p, \Lambda, q)}{\vartheta^{p-1} \omega_{j}} \exp \left(\left[-\frac{p \vartheta^{1-1 / \alpha}}{6 \alpha}\right] \omega_{j}^{-1 / \alpha}\right),
$$

then this quantity can be made smaller than one by choosing $\vartheta$ further small. Note that when we decrease the value of $\tau$ in what follows, we shall decrease also the value of $\vartheta$ accordingly.

Case 2. Initial boundary. Suppose that $\overline{Q_{\text {int }}\left(\widetilde{R}_{j}, \omega_{j}\right)}$ touches the initial boundary, that is, $t_{j+1} \leq \tilde{M} \omega_{j}^{(2-p)(1+1 / \tilde{\alpha})} \widetilde{R}_{j}^{p}$. We define

$$
Q_{\mathrm{ini}}\left(\hat{R}_{j}, \omega_{j}\right):=\left(B_{\hat{R}_{j}}\left(x_{0}\right) \cap \Omega\right) \times\left(0, \omega_{j}^{2-p} \hat{R}_{j}^{p}\right)
$$

and we assume that

$$
\sup _{\bar{Q}^{j} \cap \partial_{p} \Omega_{T}} \tilde{g} \leq \sup _{Q_{\mathrm{ini}}\left(\hat{R}_{j}, \omega_{j}\right)} v-\frac{\omega_{j}}{8} \Longrightarrow \sup _{B_{\hat{R}_{j}} \cap \Omega} v(\cdot, 0) \leq \sup _{Q_{\mathrm{ini}}\left(\hat{R}_{j}, \omega_{j}\right)} v-\frac{\omega_{j}}{8}
$$

holds. We are thus in a position to apply Lemma 3.5 and to subsequently infer (3.34):

$$
\sup _{\left(B_{\hat{R}_{j} / 4} \cap \Omega\right) \times\left(0, \omega_{j}^{2-p} \hat{R}_{j}^{p}\right)} v \leq \sup _{Q_{\mathrm{ini}}\left(\hat{R}_{j}, \omega_{j}\right)} v-\varepsilon_{4} \omega_{j} \leq \sup _{Q^{j}} v-\varepsilon_{4} \omega_{j}
$$

the last inequality holds, since $\hat{R}_{j} \leq R_{j}$ and $\omega_{j}^{2-p} \leq \widetilde{\omega}_{j}^{1-p}$. Since $R_{j+1} \leq \frac{1}{4} \hat{R}_{j}$ and

$$
t_{j+1} \leq \tilde{M} \omega_{j}^{(2-p)(1+1 / \tilde{\alpha})} \widetilde{R}_{j}^{p} \leq \omega_{j}^{2-p} \hat{R}_{j}^{p}
$$

for $\vartheta$ small, we also have $Q^{j+1} \subset\left(B_{\hat{R}_{j} / 4}\left(x_{0}\right) \cap \Omega\right) \times\left(0, \omega_{j}^{2-p} \hat{R}_{j}^{p}\right)$. Note indeed that

$$
\begin{aligned}
\frac{\tilde{M} \omega_{j}^{(2-p)(1+1 / \tilde{\alpha})}}{\omega_{j}^{2-p}}\left(\frac{\widetilde{R}_{j}}{\hat{R}_{j}}\right)^{p} & \leq \tilde{M} \omega_{j}^{(2-p) / \tilde{\alpha}} \exp \left(-\frac{\vartheta}{3 \alpha}\left[\vartheta \omega_{j}\right]^{-1 / \alpha}\right) \\
& \leq \tilde{M} \vartheta^{(p-2) / \tilde{\alpha}} \sup _{\varsigma>0} \varsigma^{(2-p) / \tilde{\alpha}} \exp \left(-\frac{\varsigma^{(\alpha-1) / \alpha}}{3 \alpha}\right) \leq 1
\end{aligned}
$$

for small enough $\vartheta$. Thus,

$$
\sup _{Q^{j+1}} v \leq \sup _{Q^{j}} v-\varepsilon_{4} \omega_{j} \quad \Longrightarrow \quad \underset{Q^{j+1}}{\operatorname{Osc}} v \leq \underset{Q^{j}}{\operatorname{osc}} v-\varepsilon_{4} \omega_{j} \leq \omega_{j}\left(1-\varepsilon_{4}\right) \leq \omega_{j+1},
$$

after having subtracted from both sides $\inf _{Q^{j+1}} v$ and taking $\vartheta \leq \varepsilon_{4}$. The case

$$
\inf _{\bar{Q}^{j} \cap \partial_{p} \Omega_{T}} \tilde{g} \geq \inf _{Q_{\mathrm{ini}}\left(\hat{R}_{j}, \omega_{j}\right)} v+\frac{\omega_{j}}{8}
$$

can be reduced to the previous one simply observing that $-v$ satisfies an equation structurally similar to (3.1) with $-\tilde{g}$ replacing $\tilde{g}$ as boundary datum; thus also in this case we conclude with (4.8). To conclude, note that we may assume that $\operatorname{osc}_{Q^{j+1}} v>$ $\frac{3}{4} \omega_{j}$, because otherwise

$$
\underset{Q^{j+1}}{\operatorname{OSc}} v \leq \frac{3}{4} \omega_{j} \leq \omega_{j+1}
$$

Copyright $\odot$ by SIAM. Unauthorized reproduction of this article is prohibited. 
Thus, if neither (4.7) nor (4.9) holds, subtracting the converse inequalities gives

$$
\underset{\bar{Q}^{j} \cap \partial_{p} \Omega_{T}}{\operatorname{Osc}} \tilde{g} \geq \underset{Q_{\mathrm{ini}}\left(\hat{R}_{j}, \omega_{j}\right)}{\operatorname{osc}} v-\frac{\omega_{j}}{4} \geq \underset{Q^{j+1}}{\operatorname{osc}} v-\frac{\omega_{j}}{4} \geq \frac{\omega_{j}}{2}
$$

in view of (4.3) this implies $\operatorname{osc}_{Q^{j+1}} v \leq \operatorname{osc}_{Q^{j}} v \leq 2 \operatorname{osc}_{\bar{Q}^{j} \cap \partial_{p} \Omega_{T}} \tilde{g}$.

Case 3. Lateral boundary. We finally assume that $B_{\widetilde{R}_{j}}\left(x_{0}\right) \cap \partial \Omega \neq \emptyset$. The idea is to use the results of section 3.1 with $\omega=\omega_{j}, r=\hat{R}_{j}$, and $\varepsilon_{1}=\tau$, which yield

$$
\widetilde{\omega}=\widetilde{\omega}_{j}, \quad T^{1}=\left(\tau \omega_{j}\right)^{2-p} \hat{R}_{j}^{p}, \quad T^{3}=\widetilde{\omega}_{j}^{1-p} \hat{R}_{j}^{p} .
$$

Since $x_{0}$ is close to the boundary, we find $\widetilde{x} \in \partial \Omega$ such that $\left|x_{0}-\widetilde{x}\right| \leq \widetilde{R}_{j}$, and thus for a small $\vartheta$ we have $B_{R_{j+1}}\left(x_{0}\right) \subset \frac{1}{16} B_{\hat{R}_{j}}(\widetilde{x})$ using (4.5). Moreover, we estimate

$$
\begin{aligned}
\frac{2 T_{j+1}}{\frac{1}{16} T^{1}} & =32\left(\frac{\omega_{j}}{\omega_{j+1}}\right)^{p-2} \frac{1}{\tau \omega_{j+1}} \exp \left((p-1)\left[\tau \omega_{j+1}\right]^{-1 / \alpha}\right)\left(\frac{R_{j+1}}{\hat{R}_{j}}\right)^{p} \\
& \leq 2^{p+3} \exp \left(\left(\left(\frac{2}{\tau}\right)^{1 / \alpha}-\frac{2}{3 \alpha} \vartheta^{(\alpha-1) / \alpha}\right) p \omega_{j}^{-1 / \alpha}\right) \leq 1
\end{aligned}
$$

for small enough $\vartheta$ using $\omega_{j} \leq 2 \omega_{j+1}$ (implied by (4.1)) and $1 / x \leq \exp \left(x^{-1 / \alpha}\right)$ for $x>0$; recall that $\alpha \in(0,1)$. Therefore $Q^{j+1} \subset \frac{1}{16} Q^{1}(\widetilde{x})$, where

$$
Q^{1}(\widetilde{x}):=\left(B_{\hat{R}_{j}}(\widetilde{x}) \times\left(t_{j+1}-T^{1}, t_{j+1}\right)\right) \cap \Omega_{T} ;
$$

moreover, we clearly have $Q^{3}(\widetilde{x}) \subset Q^{j}$ for small $\vartheta$, if we set

$$
Q^{3}(\widetilde{x}):=\left(B_{\hat{R}_{j}}(\widetilde{x}) \times\left(t_{j+1}-T^{3}, t_{j+1}\right)\right) \cap \Omega_{T} .
$$

Now we assume that

$$
\sup _{\overline{Q^{3}}(\widetilde{x}) \cap \partial_{p} \Omega_{T}} \tilde{g} \leq \sup _{Q^{3}(\widetilde{x})} v-\frac{\omega_{j}}{8} .
$$

Possibly reducing the value of $\vartheta$ and noting that the map $\sigma \mapsto \exp \left(-\sigma^{-1 / \alpha}\right)$ is increasing, (3.29) gives

$$
\underset{\frac{1}{16} Q^{1}(\widetilde{x})}{\operatorname{OSC}} v \leq \underset{Q^{3}(\widetilde{x})}{\operatorname{OSC}} v-\vartheta \omega_{j} \exp \left(-\left[\vartheta \omega_{j}\right]^{-1 / \alpha}\right) ;
$$

note that we are assuming $\varepsilon \leq \widetilde{\omega}_{j} / 2$. Using (4.3) and (4.1), we can bound the righthand side of (4.10) by $\omega_{j+1}$, which gives the result. The case

$$
\inf _{\overline{Q^{3}}(\widetilde{x}) \cap \partial_{p} \Omega_{T}} \tilde{g} \geq \inf _{Q^{3}(\widetilde{x})} v+\frac{\omega_{j}}{8}
$$

is handled similarly; see Remark 3.4. In the remaining case we have, similarly to Case 2, that either

$$
\underset{Q^{j+1}}{\operatorname{OSc}} v \leq \frac{3}{4} \omega_{j} \leq \omega_{j+1}
$$

or

$$
\underset{Q^{j+1}}{\operatorname{Osc}} v \leq \underset{Q^{j}}{\operatorname{OSc}} v \leq \omega_{j} \leq 2 \underset{\overline{Q^{3}}(\widetilde{x}) \cap \partial_{p} \Omega_{T}}{\operatorname{OSc}} \tilde{g} .
$$

This concludes the proof of (4.4); $\vartheta$ and $\tau$ are now fixed as constants depending only on $n, p, \Lambda, \delta$, and $q$. 
Lemma 4.2. Once fixed $R_{0}>0$ and $\alpha$ as in (1.6), with $\omega(\cdot)$ defined in (1.7), $\vartheta$ fixed in Proposition 4.1, $\lambda_{0}:=\exp \left(\exp \left(\vartheta^{-1 / \alpha}\right)\right)$, and $R_{j}$ defined in (4.2), the sequence $\left\{\omega_{j}\right\}_{j \in \mathbb{N}_{0}}$ with the choice $\omega_{j}:=\omega\left(R_{j}\right)$ satisfies (4.1); that is,

$$
\omega\left(R_{0}\right)=1, \quad \omega\left(R_{j+1}\right) \geq \omega\left(R_{j}\right)\left(1-\vartheta \exp \left(-\left[\vartheta \omega\left(R_{j}\right)\right]^{-1 / \alpha}\right)\right) .
$$

Moreover,

$$
\omega\left(R_{j}\right) \leq 2 \omega\left(R_{j+1}\right) .
$$

Proof. First, we obviously have $\omega\left(R_{0}\right)=1$ by the choice of $\lambda_{0}$. For any fixed $j \in \mathbb{N}_{0}$, using the elementary inequality $1-x \leq e^{-x}$ that is valid for any $x$, we have

$$
1-\vartheta \exp \left(-\left[\vartheta \omega\left(R_{j}\right)\right]^{-1 / \alpha}\right) \leq \exp \left(-\vartheta \exp \left(-\left[\vartheta \omega\left(R_{j}\right)\right]^{-1 / \alpha}\right)\right) .
$$

Now we estimate the argument of the exponential in the following way:

$$
-\vartheta \exp \left(-\left[\vartheta \omega\left(R_{j}\right)\right]^{-1 / \alpha}\right) \leq-\frac{\vartheta}{\int_{R_{j+1}}^{R_{j}} \frac{d \rho}{\rho}} \int_{R_{j+1}}^{R_{j}} \exp \left(-[\vartheta \omega(\rho)]^{-1 / \alpha}\right) \frac{d \rho}{\rho}
$$

since the map $\rho \mapsto-\exp \left(-[\vartheta \omega(\rho)]^{-1 / \alpha}\right)$ is decreasing. We compute, using the expression in (4.2) for $R_{j+1} / R_{j}$,

$$
\int_{R_{j+1}}^{R_{j}} \frac{d \rho}{\rho}=-\log \left(\frac{R_{j+1}}{R_{j}}\right)=\frac{\vartheta}{\alpha}\left[\vartheta \omega\left(R_{j}\right)\right]^{-1 / \alpha}
$$

and using (1.7) for the explicit expression of $\omega(\cdot)$

$$
\exp \left(-[\vartheta \omega(\rho)]^{-1 / \alpha}\right)=\exp \left(-\log \left(\log \left(\frac{\lambda_{0} R_{0}}{\rho}\right)\right)\right)=\frac{1}{\log \left(\lambda_{0} R_{0} / \rho\right)} .
$$

Thus, merging the estimates above and using again the aforementioned monotonicity and the expression for $\omega(\cdot)$, we have

$$
\begin{aligned}
1-\vartheta \exp \left(-\left[\vartheta \omega\left(R_{j}\right)\right]^{-1 / \alpha}\right) & \leq \exp \left(-\alpha\left[\vartheta \omega\left(R_{j}\right)\right]^{1 / \alpha} \int_{R_{j+1}}^{R_{j}} \frac{1}{\log \left(\lambda_{0} R_{0} / \rho\right)} \frac{d \rho}{\rho}\right) \\
& \leq \exp \left(-\alpha \int_{R_{j+1}}^{R_{j}}[\vartheta \omega(\rho)]^{1 / \alpha} \frac{1}{\log \left(\lambda_{0} R_{0} / \rho\right)} \frac{d \rho}{\rho}\right) \\
& =\exp \left(-\alpha \int_{R_{j+1}}^{R_{j}} \frac{1}{\log \left(\log \left(\lambda_{0} R_{0} / \rho\right)\right)} \frac{1}{\log \left(\lambda_{0} R_{0} / \rho\right)} \frac{d \rho}{\rho}\right) .
\end{aligned}
$$

We conclude by simply computing

$$
-\alpha \int_{R_{j+1}}^{R_{j}} \frac{1}{\log \left(\log \left(\lambda_{0} R_{0} / \rho\right)\right)} \frac{1}{\log \left(\lambda_{0} R_{0} / \rho\right)} \frac{d \rho}{\rho}=\log \left(\left[\frac{\log \left(\log \left(\lambda_{0} R_{0} / R_{j}\right)\right)}{\log \left(\log \left(\lambda_{0} R_{0} / R_{j+1}\right)\right)}\right]^{\alpha}\right)
$$

and since the last quantity is equal to $\log \left(\omega\left(R_{j+1}\right) / \omega\left(R_{j}\right)\right)$, the first part of the lemma is proved. For the doubling property (4.11), it is enough to recall that $\vartheta, \omega\left(R_{j}\right) \leq 1$. 
4.2. Uniform modulus of continuity. We finally prove that our approximate solution $v$ is almost equicontinuous. In order to fix a normalization condition, we assume that

$$
\underset{\Omega_{T}}{\operatorname{Osc} v \leq 1}
$$

holds true.

Proposition 4.3. Suppose that $v$ is a weak solution to (3.1) in $\Omega_{T}$ attaining continuously the boundary values $\tilde{g} \in C^{0}\left(\partial_{p} \Omega_{T}\right)$ on $\partial_{p} \Omega_{T}$, and suppose that (4.12) holds true. There is a modulus of continuity $\bar{\omega}$ independent of $\varepsilon$ and $\left(x_{0}, t_{0}\right) \in \bar{\Omega}_{T}$ such that

$$
\underset{Q_{r}\left(x_{0}, t_{0}\right) \cap \bar{\Omega}_{T}}{\mathrm{OSC}} v \leq \bar{\omega}(r)+h(\varepsilon)
$$

for every $r>0$, where $h(\varepsilon) \rightarrow 0$ as $\varepsilon \rightarrow 0$.

Proof. Fix $\left(x_{0}, t_{0}\right) \in \bar{\Omega}_{T}$. We first examine the case $r \leq r_{\Omega}^{2}$ and look for a modulus of continuity $\bar{\omega}(\cdot)$ of the form

$$
\bar{\omega}(r):=4 c_{0}\left(\omega_{1}(\sqrt{r})+\omega_{\tilde{g}}(\sqrt{r})\right),
$$

where $c_{0}>1$ is to be chosen, $\omega_{1}(\cdot)$ is $\omega(\cdot)$ defined in (1.7) with $R_{0}=1$, and $\omega_{\tilde{g}}(\cdot)$ is a modulus of continuity for $\tilde{g}$, as described in (1.4). The error $h(\cdot)$ is defined as the unique solution to

$$
\tau h(\varepsilon) \exp \left(-[\tau h(\varepsilon)]^{-1 / \alpha}\right)=2 \varepsilon,
$$

and it is easy to see that $h(\varepsilon) \rightarrow 0$ as $\varepsilon \rightarrow 0$.

According to Proposition 4.1, we define inductively

$$
\begin{aligned}
& R_{0}:=\sqrt{r}, \quad \omega_{j}:=\omega\left(R_{j}\right), \quad \widetilde{\omega}_{j}:=\tau \omega_{j} \exp \left(-\left[\tau \omega_{j}\right]^{-1 / \alpha}\right), \\
& R_{j+1}:=\exp \left(-\frac{\vartheta}{\alpha}\left[\vartheta \omega_{j}\right]^{-1 / \alpha}\right) R_{j}, \\
& Q^{j}:=\left(B_{R_{j}}\left(x_{0}\right) \times\left(t_{0}-\widetilde{\omega}_{j}^{1-p} R_{j}^{p}, t_{0}+\widetilde{\omega}_{j}^{1-p} R_{j}^{p}\right)\right) \cap \bar{\Omega}_{T} .
\end{aligned}
$$

Here $\omega(\cdot)$ is defined as in (1.7) with the expression of $R_{0}$ above. We then fix $\bar{\jmath}$ as the largest index $j$ for which $\varepsilon \leq \widetilde{\omega}_{j} / 2$; then $\omega_{j}<h(\varepsilon)$ for all $j \geq \bar{\jmath}+1$. Moreover we let $k \in \mathbb{N}_{0}$ be such that $R_{k+1} \leq r<R_{k}$ and denote $Q:=Q_{r}\left(x_{0}, t_{0}\right) \cap \bar{\Omega}_{T}$. Finally, note that if $\operatorname{osc}_{Q} v \leq 4 \omega_{\tilde{g}}\left(c_{0} \sqrt{r}\right)$, then there is nothing to prove, by the concavity of $\omega_{\tilde{g}}$. Thus we may assume that $\operatorname{osc}_{Q} v>4 \omega_{\tilde{g}}\left(c_{0} \sqrt{r}\right)$ in the rest of the proof.

We proceed inductively, showing that

$$
\underset{Q^{j}}{\operatorname{OSc} v} \leq \omega_{j}
$$

for all $j \in\{0, \ldots, \min \{\bar{\jmath}+1, k+1\}\}$. Note that (4.16) is certainly true for $j=0$ in view of (4.12), since $\omega_{0}=1$. Assume that (4.16) holds for some $j \in\{0, \ldots, \min \{\bar{\jmath}, k\}\}$. Since

$$
\omega_{j} \geq \underset{Q^{j}}{\operatorname{osc}} v>\underset{Q}{\operatorname{osc}} v>4 \omega_{\tilde{g}}\left(c_{0} \sqrt{r}\right)
$$

Copyright $@$ by SIAM. Unauthorized reproduction of this article is prohibited. 
by the fact that $R_{j} \geq R_{k} \geq r$, we see, by the doubling property $\omega_{j} \leq 2 \omega_{j+1}$ given by Lemma 4.2, that

$$
2_{\bar{Q}^{j} \cap \partial_{p} \Omega_{T}}^{\mathrm{OSC}} \tilde{g} \leq 2 \omega_{\tilde{g}}\left(c_{0} \sqrt{r}\right) \leq \frac{1}{2} \omega_{j} \leq \omega_{j+1}
$$

The first inequality follows by choosing $c_{0}=\widetilde{\omega}_{0}^{(1-p) / p}, \widetilde{\omega}_{0} \equiv \widetilde{\omega}_{0}(n, p, \Lambda, q)$, since clearly

$$
\widetilde{\omega}_{j}^{1-p} R_{j}^{p} \leq \widetilde{\omega}_{0}^{1-p} R_{0}^{p}=\widetilde{\omega}_{0}^{1-p}(\sqrt{r})^{p}=\left(c_{0} \sqrt{r}\right)^{p} .
$$

Thus, since $j \leq \bar{\jmath}$ and therefore $\widetilde{\omega}_{j} \geq 2 \varepsilon$, by Proposition 4.1 and Lemma 4.2 (note that $R_{0} \leq r_{\Omega}$ ), we obtain

$$
\underset{Q^{j+1}}{\operatorname{OSc}} v \leq \max \left\{\omega_{j+1}, 2 \underset{\bar{Q}^{j} \cap \partial_{p} \Omega_{T}}{\operatorname{osc}} \tilde{g}\right\}=\omega_{j+1},
$$

proving the induction step.

Now, if we have $\bar{\jmath} \geq k$, then (4.16) holds in particular for $j=k$. If on the other hand $\bar{\jmath}<k$ we use (4.16) with $j=\bar{\jmath}+1$; in this case, we have

$$
\underset{Q^{k}}{\operatorname{Osc} v} \underset{Q^{j+1}}{\operatorname{OSc}} v \leq \omega_{\bar{\jmath}+1}<h(\varepsilon) .
$$

As a consequence, merging the two cases, we get

$$
\underset{Q_{r}\left(x_{0}, t_{0}\right) \cap \Omega_{T}}{\operatorname{OSc}} v \leq \underset{Q^{k}}{\operatorname{Osc}} v \leq \omega_{k}+h(\varepsilon) \leq 2 \omega\left(R_{k+1}\right)+h(\varepsilon) \leq 2 \omega(r)+h(\varepsilon)
$$

and this essentially finishes the proof, since by the definition of $R_{0}$ in $\omega(\cdot), \omega(r)=$ $\omega_{1}(\sqrt{r})$. On the other hand, if $r>r_{\Omega}^{2}$, then by (4.12)

$$
\underset{Q_{r}\left(x_{0}, t_{0}\right) \cap \bar{\Omega}_{T}}{\operatorname{osc}} v \leq \frac{r}{r_{\Omega}^{2}} \leq c \omega_{1}(\sqrt{r}) .
$$

We can also give a quantified version of the previous result. Set, for $r>0$

$$
\begin{aligned}
& \widetilde{\omega}(r):=\tau \omega(r) \exp \left(-[\tau \omega(r)]^{-1 / \alpha}\right), \\
& \widetilde{Q}_{r}^{\omega(\cdot)}\left(x_{0}, t_{0}\right):=\left(B_{r}\left(x_{0}\right) \times\left(t_{0}-\widetilde{\omega}(r)^{1-p} r^{p}, t_{0}+\widetilde{\omega}(r)^{1-p} r^{p}\right)\right) \cap \bar{\Omega}_{T} .
\end{aligned}
$$

Proposition 4.4. Suppose that $v$ is a weak solution $v$ to (3.1) as in Proposition 4.3, $R_{0}>0$, and moreover that the boundary value function $\tilde{g}$ has the "intrinsic" modulus of continuity

$$
\widetilde{Q}_{r}^{\omega(\cdot)}{ }_{\left(x_{0}, t_{0}\right)}^{\operatorname{Osc}} \tilde{g} \leq \tilde{M} \omega(r)
$$

for any $r \leq R_{0}$, with some $\tilde{M}>0$, where $\left(x_{0}, t_{0}\right) \in \bar{\Omega}_{T}$ and $\omega(\cdot)$ has been defined in (1.7). Then

$$
\underset{\widetilde{Q}_{r}^{\omega(\cdot)}\left(x_{0}, t_{0}\right)}{\operatorname{osc}} v \leq c(p, \tilde{M})\left[\omega(r)+h\left(\frac{\varepsilon}{4 \tilde{M}+1}\right)\right]
$$

for any $r>0$ with $h(\varepsilon) \rightarrow 0$ as $\varepsilon \rightarrow 0$.

Copyright $@$ by SIAM. Unauthorized reproduction of this article is prohibited. 
Proof. We can rescale $v$, solution to (3.1), as we rescaled the solution to (2.4) in subsection 2.3, with $\lambda:=4 \tilde{M}+1$. Then (4.19) implies

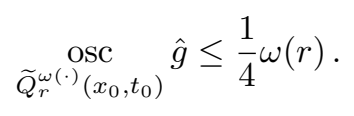

Going back to (4.17) in the proof of Proposition 4.3 we see that $2 \operatorname{osc}_{Q_{j}} \hat{g} \leq \omega_{j+1}$ is always true due to the condition (4.19) and the definitions in (4.15) (in particular, $\left.\omega_{j}:=\omega\left(R_{j}\right)\right)$. We are still in position to apply Proposition 4.3 to the solution $\hat{v}$ of (2.4) since $\int_{\mathbb{R}} \tilde{H}^{\prime}(\sigma) d \sigma=\lambda^{-1} \leq 1$; indeed, all the proofs of section 3 are based only on the properties in (2.2) of $H_{b, \varepsilon}$. Thus we obtain (4.16) for any $j \in \mathbb{N}_{0}$ and then (4.18) for $\hat{v}$ using an argument analogous to the one in the proof of Proposition 4.3. Scaling back to $v$ gives (4.20).

5. The convergence proof. In this section we conclude the proof of our main theorems. We first show that our approximants converge to a continuous function which is a physical solution of the problem thus proving Theorem 1.1. Then we see that the solution we built has the modulus of continuity (1.7), which gives Theorem 1.2 .

5.1. The Ascoli-Arzelà-type argument. We recall that $u_{\varepsilon}$ solves the regularized Cauchy-Dirichlet probem

$$
\begin{cases}\partial_{t}\left[\beta\left(u_{\varepsilon}\right)+H_{a, \varepsilon}\left(\beta\left(u_{\varepsilon}\right)\right)\right]-\operatorname{div} \mathcal{A}\left(x, t, u_{\varepsilon}, D u_{\varepsilon}\right)=0, & \text { in } \Omega_{T}, \\ u_{\varepsilon}=g & \text { on } \partial_{p} \Omega_{T},\end{cases}
$$

where the regularization of $H_{a}$ has been defined in (2.1).

Note that by the maximum principle we have

$$
\sup _{\Omega_{T}}\left|u_{\varepsilon}\right| \leq \sup _{\partial_{p} \Omega_{T}}|g|
$$

independently of $\varepsilon$; moreover $u_{\varepsilon}$ is continuous up to the boundary and it has an "equialmost-uniform" modulus of continuity in the following sense: there exists a modulus of continuity $\bar{\omega}:[0, \infty) \rightarrow[0, \infty)$, concave and continuous, such that $\bar{\omega}(0)=0$ and for every $z, z^{\prime} \in \bar{\Omega}_{T}$ and $\varepsilon \in(0,1]$ it holds that

$$
\left|u_{\varepsilon}(z)-u_{\varepsilon}\left(z^{\prime}\right)\right| \leq \bar{\omega}\left(\left|z-z^{\prime}\right|\right)+h(\varepsilon),
$$

the function $h(\cdot)$ having the property that it vanishes as $\varepsilon \rightarrow 0$.

To prove (5.2), first we define $w_{\varepsilon}=\beta\left(u_{\varepsilon}\right)$ as in subsection 2.2. Then we further rescale as in subsection 2.3 with $\left(x_{0}, t_{0}\right)=(0,0)$ and $\lambda:=\max \left\{\beta\left(2\|g\|_{L^{\infty}}\right), 1\right\}$; call the rescaled function $v$ instead of $\hat{v}$. In fact $v$ solves (3.1) with $b=a / \lambda$ and $\Omega_{T}$ replaced by $\Omega \times\left(0, \lambda^{p-2} T\right)$, and the normalization condition (4.12) is clearly satisfied. We can thus make use of Proposition 4.3, say, and this in turn yields

$$
\left|v(z)-v\left(z^{\prime}\right)\right| \leq \bar{\omega}\left(\left|z-z^{\prime}\right|\right)+h(\varepsilon)
$$

for some modulus of continuity $\bar{\omega}(\cdot)$; we can take for $\left|z-z^{\prime}\right|$ the Euclidean distance in $\mathbb{R}^{n+1}$ without loss of generality, by suitably modifying $\bar{\omega}(\cdot)$. Note that we have to use (5.1) too. This in view of the Lipschitz regularity of $\beta$ yields (5.2), where we avoided relabeling the quantities on the right-hand side. 
Call now $u_{i}, i \in \mathbb{N}$, the function $u_{\varepsilon_{i}}$ for the choice $h\left(\varepsilon_{i}\right) \leq 1 / i$; the sequence $\left\{u_{i}\right\}$ is equibounded thanks to (5.1) and, taking into account (5.2), satisfies

$$
\left|u_{i}(z)-u_{i}\left(z^{\prime}\right)\right| \leq \bar{\omega}\left(\left|z-z^{\prime}\right|\right)+1 / i
$$

for any $z, z^{\prime} \in \bar{\Omega}_{T}$. If we consider the numerable dense subset $\mathcal{S}:=\bar{\Omega}_{T} \cap \mathbb{Q}^{n+1}$, by a standard diagonal argument, as a consequence of (5.1), we extract a subsequence, still denoted by $\left\{u_{i}\right\}_{i \in \mathbb{N}}$, converging pointwise in $\mathcal{S}$ to $u$. Moreover, slightly modifying the proof of Ascoli-Arzelà (see, for instance, the proof given in [7, p. 17]), using condition (5.3) instead of equicontinuity, we show that the sequence $\left\{u_{i}\right\}_{i \in \mathbb{N}}$ actually converges pointwise in $\bar{\Omega}_{T}$ to a function which we shall call $u$, and moreover, by a similar argument, the convergence is uniform. In particular, $u \in C^{0}\left(\bar{\Omega}_{T}\right)$. The rest of the proof will be devoted to proving that $u$ is a local weak solution of (1.2). Assuming this for a moment, we now prove Theorem 1.2.

Proof of Theorem 1.2. The constant $\vartheta$ is the one in Proposition 4.1, while $\lambda_{0}$ has been fixed in Lemma 4.2. The constant $\tilde{\delta} \in(0,1)$ is defined, according to Proposition 4.1, as

$$
\tilde{\delta}^{2-p}:=\widetilde{\omega}_{0}^{1-p}=\tau^{1-p} \exp \left((p-1) \tau^{-1 / \alpha}\right) .
$$

To prove (1.9) we distinguish two cases. If $\omega_{0} \leq 1$, then $(1.8)_{1}$ directly implies that $\operatorname{osc}_{\tilde{Q}} u \leq 1$ with $\tilde{Q}:=\left(B_{R_{0}}\left(x_{0}\right) \times\left(t_{0}-\tilde{\delta}^{2-p} R_{0}^{p}, t_{0}+\tilde{\delta}^{2-p} R_{0}^{p}\right)\right) \cap \Omega_{T}$ and we recognize that, by our choice of $\tilde{\delta}$, the cylinder $\tilde{Q}$ is exactly the cylinder $Q^{0}$ appearing in Proposition 4.1. In the proof of Proposition 4.3 we can clearly replace the renormalization in (4.12) with this local information, which is sufficient to start the iteration. On the other hand, jumping to Proposition 4.4, we note that for $\gamma \in(0,1)$ as in $(1.8)_{2}$, $\widetilde{\omega}(r)^{1-p} \leq c_{\gamma}\left(r / R_{0}\right)^{-p \gamma}, c_{\gamma}$ depending on data, $\gamma$, and $R_{0}$, so

$$
\begin{aligned}
\underset{\tilde{Q}_{r}^{(\cdot)}\left(x_{0}, t_{0}\right)}{\operatorname{OSc}} \tilde{g} & \leq \underset{B_{r}\left(x_{0}\right) \times\left(t_{0}-c_{\gamma}\left(r / R_{0}\right)^{p(1-\gamma)}, t_{0}+c_{\gamma}\left(r / R_{0}\right)^{p(1-\gamma)}\right)}{\operatorname{Osc}} \tilde{g} \\
& \leq c\left(c_{\gamma}, R_{0}\right) \tilde{M} \omega_{g}\left(\left(r / R_{0}\right)^{1-\gamma}\right) \leq c \omega(r),
\end{aligned}
$$

and (4.19) is satisfied. Thus we have (4.20) at hand for $v=\beta\left(w_{1 / i}\right)$ and, after passing to the limit as $\varepsilon=1 / i \searrow 0$, we infer (1.9).

In the case $\omega_{0}>1$, the proof is exactly the same except for the fact that before starting we rescale $u, g$ to $\hat{u}, \hat{g}$ as in subsection 2.3 with $\lambda=\omega_{0}$. We again obtain $\operatorname{osc}_{Q^{0}} v \leq 1$ and (4.19), and we conclude by invoking (4.20) and scaling back to $u$. Note that $Q_{r}^{\omega_{0}} \supset Q_{\omega_{0}^{(2-p) / p} r}$ and (1.9) holds trivially if $r>\omega_{0}^{(2-p) / p} R_{0}$.

In the following subsection we show that the pointwise limit $u$ is a physical solution to our problem, that is, it satisfies the weak formulation of Definition 1.3.

5.2. Convergence away from the jump. We consider the previously defined sequence $\left\{u_{i}\right\}_{i \in \mathbb{N}}$ which converges uniformly in $\Omega_{T}$ to $u$. Here it is more convenient to work with $w_{i}:=\beta\left(u_{i}\right)$, which solves

$$
\partial_{t} w_{i}-\operatorname{div} \overline{\mathcal{A}}\left(x, t, w_{i}, D w_{i}\right)=-\partial_{t} H_{a, \varepsilon_{i}}\left(w_{i}\right)
$$

locally in $\Omega_{T}$, with $\overline{\mathcal{A}}$, having the same structure as $\mathcal{A}$; see (2.5). Note that also $\left\{w_{i}\right\}$ converges uniformly to $w=\beta(u)$. The reader might recall now that

$$
\operatorname{supp} H_{a, \varepsilon_{i}}^{\prime}(\cdot) \subset\left(a-\varepsilon_{i}, a+\varepsilon_{i}\right) ;
$$


hence, in the set $\Omega_{T} \cap\left\{\left|w_{i}-a\right| \geq \varepsilon_{i}\right\} w_{i}$ is a solution to a $p$-Laplacian-type equation

$$
\partial_{t} w_{i}-\operatorname{div} \overline{\mathcal{A}}\left(x, t, w_{i}, D w_{i}\right)=0 .
$$

Now we fix $\sigma>0$. By the uniform convergence, there exists $\bar{n}=\bar{n}(\sigma, \omega)$ such that

$$
\Omega_{T} \cap\{|w-a| \geq 2 \sigma\} \subset \Omega_{T} \cap\left\{\left|w_{i}-a\right| \geq \sigma\right\} \subset \Omega_{T} \cap\left\{\left|w_{i}-a\right| \geq \varepsilon_{i}\right\}
$$

for all $i \geq \bar{n} ;$ indeed

$$
\left|w_{i}(x)-a\right| \geq|w-a|-\left|w_{i}(x)-w(x)\right| \geq 2 \sigma-\sigma=\sigma
$$

if (and independently of) $x \in \Omega_{T} \cap\{|w-a| \geq 2 \sigma\}$ and if $i \geq \bar{n}$ is large enough, by the uniform convergence of $w_{i}$ to $w$. Hence $w_{i}$ is a solution to an evolutionary $p$-Laplaciantype equation in $\Omega_{T} \cap\{|w-a| \geq 2 \sigma\}$ for all $i \geq \bar{n}$. Therefore using an argument similar to the proof of [10, Theorem 5.3], we find not only that $\left\{w_{i}\right\}_{i \geq \bar{n}}$ converges to $w$ uniformly in $\Omega_{T} \cap\{|w-a| \geq 2 \sigma\}$, but also $D w_{i} \rightarrow D w$ almost everywhere in this set (and moreover $D w \in L_{\text {loc }}^{p}\left(\Omega_{T} \cap\{|w-a| \geq 2 \sigma\}\right)$ ); we shall sketch the proof in the next subsection. At this point, using a diagonal argument, we get that there exists a subsequence of the $\left\{w_{i}\right\}$ defined in subsection 5.1, still denoted by $\left\{w_{i}\right\}$, such that $w_{i}$ converges uniformly to $w$ in $\Omega_{T}$ and moreover

$$
D w_{i} \rightarrow D w \quad \text { almost everywhere in }|w-a|>0 .
$$

5.3. Almost everywhere convergence of the gradients. We give here a short proof of the statement about the almost everywhere convergence of the gradients in the previous subsection. We only give a hint of the classic proof and refer to $[2,3,10]$ for more details.

Take two concentric cylinders $\tilde{\mathcal{Q}} \Subset \mathcal{Q} \Subset \Omega_{T} \cap\{|w-a| \geq 2 \sigma\}$, two functions $w_{j}, w_{k}$ of the sequence $\left\{w_{i}\right\}$, and a "small" number $\varsigma>0$. We test, respectively, the weak formulations of (5.5) for $w_{j}$ and $w_{k}$ with the functions

$$
\varphi_{\mp}(x, t):=\left(\varsigma \mp T_{\varsigma}\left(w_{j}-w_{k}\right)\right) \phi^{p}, \quad \text { where } \quad T_{\varsigma}(s):=\min \{\max \{s,-\varsigma\}, \varsigma\}
$$

is the usual truncation function of $[2], \phi \in V_{\mathrm{loc}}^{2, p}(\mathcal{Q})$ with $\partial_{t} \phi \in L_{\mathrm{loc}}^{2}(\mathcal{Q}), \phi(\cdot, T) \equiv 0$, and $\phi \equiv 1$ in $\overline{\mathcal{Q}}$. Note that $\varphi_{\mp}$ are admissible since $s \rightarrow T_{\varsigma}(s)$ is a Lipschitz mapping and that this is actually a formal choice, due to the fact that these test functions do not have the needed time regularity. However, in [10, Proof of Theorem 5.3] it is shown how to appropriately perform this delicate double limiting procedure. Note that using the bi-Lipschitz relation (1.11) we infer, from (1.12) and (1.13), that

$$
\begin{gathered}
\langle\overline{\mathcal{A}}(x, t, u, \xi)-\overline{\mathcal{A}}(x, t, u, \zeta), \xi-\zeta\rangle>0, \\
\sup _{(x, t) \in \Omega_{T}}|\overline{\mathcal{A}}(x, t, u, \xi)-\overline{\mathcal{A}}(x, t, v, \xi)| \leq \bar{K}(M, \tilde{M}) \omega_{\overline{\mathcal{A}}, u}(|u-v|)
\end{gathered}
$$

for almost every $(x, t) \in \Omega_{T}$ and for all $(u, v, \xi, \zeta) \in \mathbb{R}^{2(n+1)}$, with $\zeta \neq \xi$ for the monotonicity condition and $|u|+|v| \leq M$ and $|\xi| \leq \tilde{M}$ for the continuity one; note that we also used the concavity of $\omega_{\mathcal{A}, \xi}$. Indeed

$$
\begin{aligned}
\omega_{\mathcal{A}, \xi}\left(|\xi|\left|\frac{1}{\beta^{\prime}\left(\beta^{-1}(u)\right)}-\frac{1}{\left.\beta^{\prime}\left(\beta^{-1}(v)\right)\right)}\right|\right) & \leq \tilde{M} \omega_{\mathcal{A}, \xi}\left(\Lambda^{2} \bar{K}(M) \omega_{\beta^{\prime}}(\Lambda|u-v|)\right) \\
& \leq \tilde{\Lambda}^{3} M \bar{K}(M) \omega_{\mathcal{A}, \xi}\left(\omega_{\beta^{\prime}}(|u-v|)\right)
\end{aligned}
$$

Copyright $@$ by SIAM. Unauthorized reproduction of this article is prohibited. 
where $\bar{K}(M) \omega_{\beta^{\prime}}$ is clearly the concave modulus of continuity for $\beta^{\prime}$ when $u, v$ vary in the compact set $|u|+|v| \leq M$. Now this choice, after some algebraic manipulations (performed in detail in the aforementioned proof), leads to

$$
\begin{aligned}
& \int_{\mathcal{Q} \cap\left\{\left|w_{j}-w_{k}\right| \leq \varsigma\right\}}\left\langle\overline{\mathcal{A}}\left(\cdot, \cdot, w_{j}, D w_{j}\right)-\overline{\mathcal{A}}\left(\cdot, \cdot, w_{k}, D w_{k}\right), D\left(w_{j}-w_{k}\right)\right\rangle \phi^{p} d x d t \\
& \leq-\int_{\mathcal{Q} \cap\left\{\left|w_{j}-w_{k}\right| \leq \varsigma\right\}}\left\langle\overline{\mathcal{A}}\left(\cdot, \cdot, w_{j}, D w_{j}\right)-\overline{\mathcal{A}}\left(\cdot, \cdot, w_{k}, D w_{k}\right), D \phi^{p}\right\rangle\left(w_{j}-w_{k}\right) d x d t \\
& \quad+c \varsigma+\varsigma \int_{\mathcal{Q} \cap\left\{\left|w_{j}-w_{k}\right| \leq \varsigma\right\}}\left\langle\overline{\mathcal{A}}\left(\cdot, \cdot, w_{j}, D w_{j}\right)+\overline{\mathcal{A}}\left(\cdot, \cdot, w_{k}, D w_{k}\right), D \phi^{p}\right\rangle d x d t \\
& \quad \leq c \varsigma,
\end{aligned}
$$

where the constant ultimately depends upon $p, \Lambda,|\mathcal{Q}|, \sup _{\partial_{p} \Omega_{T}}|g|, \phi$ (hence on $\overline{\mathcal{Q}}$ and $\mathcal{Q})$ but not on $j, k$. In the last inequality we took into account the growth condition in (2.6), the standard energy estimate for the $p$-Laplacian equation (5.5), together with the uniform bound (5.1).

The goal here is to prove that the sequence $\left\{D w_{i}\right\}_{i \in \mathbb{N}}$ converges in measure, being a Cauchy sequence with respect to this convergence. This together with the fact that the gradients are uniformly bounded in the $L^{p}$ norm - and this follows again by the Caccioppoli's estimate and (5.1) - would then lead to the needed almost everywhere convergence. To this aim we define, for $\varsigma$ as above and $\rho, \lambda>0$, the sets

$$
\begin{aligned}
& E_{j, k}^{\rho}:=\left\{z \in \tilde{\mathcal{Q}}:\left|D w_{j}(z)-D w_{k}(z)\right| \geq \rho\right\} \\
& U_{j, k}^{\varsigma}:=\left\{z \in \tilde{\mathcal{Q}}:\left|w_{j}(z)-w_{k}(z)\right| \leq \varsigma\right\} ; \\
& V_{j, k}^{\lambda}:=\left\{z \in \tilde{\mathcal{Q}}: \max \left\{\left|D w_{j}(z)\right|,\left|D w_{k}(z)\right|\right\} \leq \lambda\right\} .
\end{aligned}
$$

First note that, enlarging appropriately the domains of integration, we infer

$$
\begin{aligned}
& \int_{E_{j, k}^{\rho} \cap U_{j, k}^{\varsigma} \cap V_{j, k}^{\lambda}}\left\langle\overline{\mathcal{A}}\left(\cdot, \cdot, w_{j}, D w_{j}\right)-\overline{\mathcal{A}}\left(\cdot, \cdot, w_{j}, D w_{k}\right), D\left(w_{j}-w_{k}\right)\right\rangle \phi^{p} d x d t \\
& \leq \int_{\mathcal{Q} \cap\left\{\left|w_{j}-w_{k}\right| \leq \varsigma\right\}}\left\langle\overline{\mathcal{A}}\left(\cdot, \cdot, w_{j}, D w_{j}\right)-\overline{\mathcal{A}}\left(\cdot, \cdot, w_{k}, D w_{k}\right), D\left(w_{j}-w_{k}\right)\right\rangle \phi^{p} d x d t \\
& \quad+|\mathcal{Q}| \bar{K}\left(2\|g\|_{\left.L^{\infty}, \lambda\right) \omega_{\overline{\mathcal{A}}, u}(\varsigma)}\right. \\
& \quad \leq c\left(\varsigma+\bar{K}\left(2\|g\|_{L^{\infty}}, \lambda\right) \omega_{\overline{\mathcal{A}}, u}(\varsigma)\right)
\end{aligned}
$$

by (5.7) together with (5.1) and (5.8), for an appropriate test function equal to one on $\tilde{\mathcal{Q}}$. In order to prove that the sequence $\left\{D w_{i}\right\}_{i \in \mathbb{N}}$ is a Cauchy sequence with respect to the convergence in measure, that is, that for any $\rho>0$, once we fix $\epsilon>0$ we can find $\bar{n} \equiv \bar{n}(\epsilon)$ such that $\left|E_{j, k}^{\rho}\right| \leq \epsilon$ for all $j, k \geq \bar{n}$, we then split

$$
\left|E_{j, k}^{\rho}\right| \leq\left|E_{j, k}^{\rho} \cap U_{j, k}^{\varsigma}\right|+\left|\tilde{\mathcal{Q}} \backslash U_{j, k}^{\varsigma}\right| \leq\left|E_{j, k}^{\rho} \cap U_{j, k}^{\varsigma} \cap V_{j, k}^{\lambda}\right|+\left|\tilde{\mathcal{Q}} \backslash U_{j, k}^{\varsigma}\right|+\left|\tilde{\mathcal{Q}} \backslash V_{j, k}^{\lambda}\right|
$$

for appropriate $\varsigma, \lambda>0$ that will be chosen in the following lines. Notice now that since $\left\{w_{i}\right\}$ converges uniformly, then in particular it is a Cauchy sequence in $\tilde{\mathcal{Q}}$ and hence $\left|\tilde{\mathcal{Q}} \backslash U_{j, k}^{\varsigma}\right| \leq \epsilon / 3$ provided we take $j, k$ large enough. Moreover, since the sequence $\left\{D w_{i}\right\}$ is bounded in $L^{p}$, then $\left|\tilde{\mathcal{Q}} \backslash V_{j, k}^{\lambda}\right| \leq \epsilon / 3$ for $\lambda$ large enough; hence we can now restrict our attention to the set $E_{j, k}^{\rho} \cap U_{j, k}^{\varsigma} \cap V_{j, k}^{\lambda}$. We consider the set

$$
\mathcal{K}_{(x, t)}^{\lambda, \rho}:=\left\{(u, \xi, \zeta) \in \mathbb{R}^{2 n+1}:|u| \leq\|g\|_{L^{\infty}},|\xi|,|\zeta| \leq \lambda,|\xi-\zeta| \geq \rho\right\}
$$


(notice that $\lambda$ and $\rho$ are fixed) and we consider, for $(x, t) \in \tilde{\mathcal{Q}}$, the function

$$
\gamma(x, t):=\inf _{\mathcal{K}_{(x, t)}^{\lambda}}\langle\overline{\mathcal{A}}(x, t, u, \xi)-\overline{\mathcal{A}}(x, t, u, \zeta), \xi-\zeta\rangle ;
$$

by the continuity of $(u, \xi) \mapsto \overline{\mathcal{A}}(\cdot, \cdot, u, \xi)$, the compactness of $\mathcal{K}_{(x, t)}^{\lambda, \rho}$, and the monotonicity of $\overline{\mathcal{A}}$ in (5.7), we infer that $\gamma(x, t)>0$ for almost every $(x, t) \in \tilde{\mathcal{Q}}$. By $(5.9)$ we then have

$$
\begin{aligned}
\left|E_{j, k}^{\rho} \cap U_{j, k}^{\varsigma} \cap V_{j, k}^{\lambda}\right| f_{E_{j, k}^{\rho} \cap U_{j, k}^{\varsigma} \cap V_{j, k}^{\lambda}} \gamma d x d t & \leq \int_{E_{j, k}^{\rho} \cap U_{j, k}^{\varsigma} \cap V_{j, k}^{\lambda}} \gamma d x d t \\
& \leq c\left(\varsigma+\bar{K}\left(2\|g\|_{\left.\left.L^{\infty}, \lambda\right) \omega_{\overline{\mathcal{A}}, u}(\varsigma)\right)}\right.\right.
\end{aligned}
$$

and since $\gamma>0$ a.e. in $\tilde{\mathcal{Q}}$, we conclude with $\left|E_{j, k}^{\rho} \cap U_{j, k}^{\varsigma} \cap V_{j, k}^{\lambda}\right| \leq \epsilon / 3$ for $\varsigma$ small enough (recall $\lambda$ has been already fixed). Hence we have proved that $\left\{D w_{i}\right\}$ is a Cauchy sequence with respect to the convergence in measure. This, together with the convergence of $\left\{w_{i}\right\}$, yields that $\left\{D w_{i}\right\}$ actually converges to $D w$ in measure and hence there exists a subsequence which converge almost everywhere. Since the argument above actually holds for every subsequence, then almost everywhere convergence takes place for the full sequence $\left\{D w_{i}\right\}$. Finally, since $\tilde{\mathcal{Q}}$ is an arbitrary compactly contained subset of $\Omega_{T} \cap\{|w-a| \geq 2 \sigma\}$ and the whole sequence converges almost everywhere, we have almost everywhere convergence in the whole $\Omega_{T} \cap\{|w-a| \geq 2 \sigma\}$. The fact that $D w \in L^{p}\left(\Omega_{T} \cap\{|w-a| \geq 2 \sigma\}\right)$ now simply follows by Lebesgue's dominated convergence theorem and by the fact that the sequence $D w_{i}$ is equibounded in $L^{p}$.

5.4. Convergence near the jump. In order to infer information on the behavior of the gradient of the approximating solutions in the set close to $\{w=a\}$, we (formally) test (5.4) with the function $\varphi=T_{2 \sigma}\left(w_{i}-a\right) \phi^{p}$, for some fixed $\sigma \in(0,1)$, where $T_{2 \sigma}$ has been defined in (5.6) and $\phi \in C_{c}^{\infty}\left(\Omega_{T}\right)$. The rigorous treatment needs a mollification in time; see [1] for details. We note that since

$$
D \varphi=D w_{i} \chi_{\left\{\left|w_{i}-a\right| \leq 2 \sigma\right\}} \phi^{p}+p \phi^{p-1} T_{2 \sigma}\left(w_{i}-a\right) D \phi,
$$

we have, with $\mathcal{H}$ as in (2.7),

$$
\begin{aligned}
\int_{\Omega_{T} \cap\left\{\left|w_{i}-a\right| \leq 2 \sigma\right\}}\left|D w_{i}\right|^{p} \phi^{p} d x d t & \leq c(p, \Lambda) \sigma^{p}\|D \phi\|_{L^{p}}^{p} \\
& +c(p, \Lambda) \int_{\Omega_{T}} \int_{a}^{w_{i}} \mathcal{H}^{\prime}(\xi) T_{2 \sigma}(\xi-a) d \xi \partial_{t}\left(\phi^{p}\right) d x d t .
\end{aligned}
$$

For the parabolic term we formally have

$$
\begin{aligned}
\int_{\Omega_{T}} \partial_{t} w_{i} \mathcal{H}^{\prime}\left(w_{i}\right) & T_{2 \sigma}\left(w_{i}-a\right) \phi^{p} d x d t \\
= & \int_{\Omega_{T}} \partial_{t}\left[\int_{a}^{w_{i}} \mathcal{H}^{\prime}(\xi) T_{2 \sigma}(\xi-a) d \xi\right] \phi^{p} d x d t \\
= & \int_{\Omega}\left[\int_{a}^{w_{i}} \mathcal{H}^{\prime}(\xi) T_{2 \sigma}(\xi-a) d \xi \phi^{p}\right](\cdot, T) d x \\
& \quad-\int_{\Omega_{T}} \int_{a}^{w_{i}} \mathcal{H}^{\prime}(\xi) T_{2 \sigma}(\xi-a) d \xi \partial_{t}\left(\phi^{p}\right) d x d t .
\end{aligned}
$$

We can discard the first term since

$$
\int_{a}^{w_{i}(x, T)} \mathcal{H}^{\prime}(\xi) T_{2 \sigma}(\xi-a) d \xi \geq 0
$$

Copyright $\odot$ by SIAM. Unauthorized reproduction of this article is prohibited. 
the mapping $\xi \mapsto T_{2 \sigma}(\xi-a)$ being odd with respect to the point $\xi=a$ and $\mathcal{H}^{\prime}(\cdot) \geq 0$. Moreover,

$$
\begin{aligned}
\int_{\Omega_{T}} \int_{a}^{w_{i}} \mathcal{H}^{\prime}(\xi) & T_{2 \sigma}(\xi-a) d \xi \partial_{t}\left(\phi^{p}\right) d x d t \\
\leq & c(n, p) \sigma\left\|\partial_{t}\left(\phi^{p}\right)\right\|_{L^{1}\left(\Omega_{T}\right)}\left(\left\|w_{i}\right\|_{L^{\infty}\left(Q_{2 R}\right)}+a_{+}+1\right) \\
& \leq c(n, p) \sigma\left\|\partial_{t}\left(\phi^{p}\right)\right\|_{L^{1}\left(\Omega_{T}\right)}\left(\|\beta(g)\|_{L^{\infty}\left(\partial_{p} \Omega_{T}\right)}+a_{+}+1\right)
\end{aligned}
$$

by the fact that $\left|T_{2 \sigma}(\cdot)\right| \leq 2 \sigma$ and (5.1). Thus, also in view of (5.1), we can finally estimate

$$
\int_{\Omega_{T} \cap\left\{\left|w_{i}-a\right| \leq 2 \sigma\right\}}\left|D w_{i}\right|^{p} \phi^{p} d x d t \leq c \sigma,
$$

where $c$ depends on $n, p, \Lambda, a,\|g\|_{L^{\infty}\left(\partial_{p} \Omega_{T}\right)}, \beta$, and test function $\phi$.

5.5. Passing to the limit. We now want to pass to the limit in the weak formulation of (5.4); this, once fixed $\sigma \in(0,1), \mathcal{K} \Subset \Omega,\left[t_{1}, t_{2}\right] \subset[0, T]$ and a test function $\varphi$ as in Definition 1.3, reads as

$$
\begin{gathered}
0=\int_{\mathcal{K}_{t_{1}, t_{2}} \cap\left\{\left|w_{i}-a\right|>\sigma\right\}}\left\langle\overline{\mathcal{A}}\left(\cdot, \cdot, w_{i}, D w_{i}\right), D \varphi\right\rangle d x d t \\
-\int_{\mathcal{K}_{t_{1}, t_{2}}}\left[w_{i}+H_{a, \varepsilon_{i}}\left(w_{i}\right)\right] \partial_{t} \varphi d x d t \\
+\int_{\mathcal{K}_{t_{1}, t_{2}} \cap\left\{\left|w_{i}-a\right| \leq \sigma\right\}}\left\langle\overline{\mathcal{A}}\left(\cdot, \cdot, \cdot w_{i}, D w_{i}\right), D \varphi\right\rangle d x d t \\
\quad+\left.\int_{\mathcal{K}}\left\{\left[w_{i}+H_{a, \varepsilon_{i}}\left(w_{i}\right)\right] \varphi\right\}(\cdot, \tau) d x\right|_{\tau=t_{1}} ^{t_{2}} .
\end{gathered}
$$

By the continuity of $(\mu, \xi) \mapsto \mathcal{A}(\cdot, \cdot, \mu, \xi)$, the first term converges to

$$
\int_{\mathcal{K}_{t_{1}, t_{2}} \cap\{|w-a|>\sigma\}}\langle\mathcal{A}(\cdot, \cdot, u, D u), D \varphi\rangle d x d t
$$

as $i \rightarrow \infty$; indeed $\overline{\mathcal{A}}\left(\cdot, \cdot, w_{i}, D w_{i}\right)=\mathcal{A}\left(\cdot, \cdot, u_{i}, D u_{i}\right)$. The second and fourth terms converge to

$$
-\int_{\mathcal{K}_{t_{1}, t_{2}}} \xi \partial_{t} \varphi d x d t+\left.\int_{\mathcal{K}}[\xi \varphi](\cdot, \tau) d x\right|_{\tau=t_{1}} ^{t_{2}},
$$

where $\xi$ belongs to the graph $\beta(u)+H_{a}(\beta(u)$ ) (in particular, $\xi=1 / 2$ if $\beta(u)=0$ ). Finally, by uniform convergence we can find $\bar{n}$, depending on $\omega$ and $\sigma$, such that

$$
\mathcal{K}_{t_{1}, t_{2}} \cap\{|w-a| \leq \sigma\} \subset \mathcal{K}_{t_{1}, t_{2}} \cap\left\{\left|w_{i}-a\right| \leq 2 \sigma\right\} \quad \text { for any } i \geq \bar{n} .
$$

Hence we can bound, for $i \geq \bar{n}$,

$$
\begin{aligned}
\left|\mathcal{T}_{i, \sigma}\right|:=\left|\int_{\mathcal{K}_{t_{1}, t_{2}} \cap\{|w-a| \leq \sigma\}}\left\langle\overline{\mathcal{A}}\left(\cdot, \cdot, w_{i}, D w_{i}\right), D \varphi\right\rangle d x d t\right| \\
\leq c(\Lambda)\|D \varphi\|_{L^{p}\left(\mathcal{K}_{t_{1}, t_{2}}\right)}\left(\int_{\Omega_{T} \cap\left\{\left|w_{i}-a\right| \leq 2 \sigma\right\}}\left|D w_{i}\right|^{p} \chi_{\operatorname{supp} \varphi} d x d t\right)^{1 / p^{\prime}}
\end{aligned}
$$

Copyright $@$ by SIAM. Unauthorized reproduction of this article is prohibited. 


$$
\begin{aligned}
& \leq c\|D \varphi\|_{L^{p}\left(\mathcal{K}_{t_{1}, t_{2}}\right)}\left(\int_{\Omega_{T} \cap\left\{\left|w_{i}-a\right| \leq 2 \sigma\right\}}\left|D w_{i}\right|^{p} \phi^{p} d x d t\right)^{1 / p^{\prime}} \\
& \leq c\|D \varphi\|_{L^{p}\left(\mathcal{K}_{t_{1}, t_{2}}\right)} \sigma^{1 / p^{\prime}}
\end{aligned}
$$

by (5.10), with $c \equiv c(p, \Lambda)$; here we must take $\phi$ as an appropriate cut-off function, equal to one on the support of $\varphi$. Hence if we pass to the limit (superior) $i \rightarrow \infty$ in (5.11) with $\sigma \in(0,1)$ fixed, by rearranging terms we get

$$
\begin{aligned}
-\int_{\mathcal{K}_{t_{1}, t_{2}}} \xi \partial_{t} \varphi d x d t+\int_{\mathcal{K}_{t_{1}, t_{2}} \cap\{|\beta(u)-a|>\sigma\}}\langle\mathcal{A}(\cdot, \cdot, u, D u), D \varphi\rangle d x d t+\mathcal{T}_{\sigma} & \\
& +\left.\int_{\mathcal{K}}[\xi \varphi](\cdot, \tau) d x\right|_{\tau=t_{1}} ^{t_{2}}=0
\end{aligned}
$$

with $\xi \in \beta(u)+H_{a}(\beta(u))$, since the equibounded sequence $\mathcal{T}_{i, \sigma}$ converges to a limit (possibly only superior) $\mathcal{T}_{\sigma}$ such that $\left|\mathcal{T}_{\sigma}\right| \leq c \sigma^{1 / p^{\prime}}$ by $(5.12)$. Now we take the limit $\sigma \rightarrow 0$ and we get

$$
\begin{aligned}
-\int_{\mathcal{K}_{t_{1}, t_{2}}} \xi \partial_{t} \varphi d x d t+\int_{\mathcal{K}_{t_{1}, t_{2}} \cap\{\beta(u) \neq a\}}\langle\mathcal{A}(\cdot, \cdot, u, D u), D \varphi\rangle d x d t & \\
& +\left.\int_{\mathcal{K}}[\xi \varphi](\cdot, \tau) d x\right|_{\tau=t_{1}} ^{t_{2}}=0 .
\end{aligned}
$$

Using well-known properties of Sobolev functions and (1.11), the second integral is equal to the integral over $\mathcal{K}_{t_{1}, t_{2}}$ of the same function. Hence, we have proved that the pointwise limit defined in subsection 5.1 is a local weak solution to (1.2) in the sense of Definition 1.3.

Acknowledgments. This paper was partially conceived while the authors were part of the research program "Evolutionary Problems" at the Institut Mittag-Leffler (Djursholm, Sweden) in fall 2013. The support, the hospitality, and the optimal environment of the Institut are gratefully acknowledged. The paper was concluded after visits of the first and fourth authors to Aalto University and of the second author to the University of Coimbra. The authors are grateful to both institutions.

\section{REFERENCES}

[1] P. Baroni, T. Kuusi, and J. M. Urbano, A quantitative modulus of continuity for the twophase Stefan problem, Arch. Ration. Mech. Anal., 214 (2014), pp. 545-573.

[2] L. Boccardo AND T. Gallouët, Nonlinear elliptic and parabolic equations involving measure data, J. Funct. Anal., 87 (1989), pp. 149-169.

[3] L. Boccardo, A. Dall'Aglio, T. Gallouët, and L. Orsina, Nonlinear parabolic equations with measure data, J. Funct. Anal., 147 (1997), pp. 237-258.

[4] E. DiBenedetto, Continuity of weak solutions to certain singular parabolic equations, Ann. Mat. Pura Appl. (4), 103 (1982), pp. 131-176.

[5] E. DiBenedetto, A boundary modulus of continuity for a class of singular parabolic equations, J. Differential Equations, 63 (1986), pp. 418-447.

[6] E. DiBenedetto, Degenerate Parabolic Equations, Universitext, Springer-Verlag, New York, 1993.

[7] E. DiBenedetto, Partial Differential Equations, Birkhäuser Boston, Boston, MA, 1995.

[8] E. DiBenedetto, J. M. Urbano, and V. Vespri, Current issues on singular and degenerate evolution equations, in Evolutionary Equations. Vol. I, Handb. Differ. Equ., North-Holland, Amsterdam, 2004, pp. 169-286. 
[9] L. C. Evans and R. F. Gariepy, Measure Theory and Fine Properties of Functions, Stud. Adv. Math., CRC Press, Boca Raton, FL, 1992.

[10] R. Korte, T. KuUsi, and M. Parviainen, A connection between a general class of superparabolic functions and supersolutions, J. Evol. Equ., 10 (2010), pp. 1-20.

[11] T. Kuusi And G. Mingione, The Wolff gradient bound for degenerate parabolic equations, J. Eur. Math. Soc. (JEMS), 16 (2014), pp. 835-892.

[12] R. H. Nochetto, A class of nondegenerate two-phase Stefan problems in several space variables, Comm. Partial Differential Equations, 12 (1987), pp. 21-45.

[13] S. SAlSA, Two-phase Stefan problem. Recent results and open questions, Milan J. Math., 80 (2012), pp. 267-281.

[14] J. M. URbano, Continuous solutions for a degenerate free boundary problem, Ann. Mat. Pura Appl. (4), 178 (2000), pp. 195-224.

[15] J. M. Urbano, The Method of Intrinsic Scaling, A Systematic Approach to Regularity for Degenerate and Singular PDEs, Lecture Notes in Math. 1930, Springer-Verlag, Berlin, 2008.

[16] W. P. Ziemer, Interior and boundary continuity of weak solutions of degenerate parabolic equations, Trans. Amer. Math. Soc., 271 (1982), pp. 733-748.

Copyright (c) by SIAM. Unauthorized reproduction of this article is prohibited. 